\title{
Origin of attraction, magnitude and directionality of interactions in benzene complexes with
}

\section{pyridinium cations}

\author{
Seiji Tsuzuki, ${ }^{*}{ }^{\dagger}$ Masuhiro Mikamit'and Shinji Yamada,
}

National Institute of Advanced Industrial Science and Technology (AIST), Tsukuba, Ibaraki 305-8568, Japan, and Department of Chemistry, Faculty of Science, Ochanomizu University, Bunkyo-Ku, Tokyo 112-8610

$\dagger$ AIST,

\$ Ochanomizu University

\section{Supporting Information}

Discussion on the basis set and electron correlation effects, and the estimation procedure of the MP2 and $\operatorname{CCSD}(\mathrm{T})$ interaction energies at the basis set limit.

\section{Basis set and electron correlation correction effects}

Intermolecular interaction energies for the benzene complexes with pyridine, pyridinium and $N$-methylpyridinium (1f, 2g and $\mathbf{3 f}$ in Figs. 5, 7 and 9) were calculated at the HF and MP2 levels using several basis sets to evaluate the basis set effects. The basis set dependence of the HF interaction energy $\left(\mathrm{E}_{\mathrm{HF}}\right)$ is small, while the MP2 interaction energy ( $\mathrm{E}_{\mathrm{MP}}$ ) 
depends substantially on the basis set (Table 1S). Small basis sets underestimate the attraction as in the cases of other aromatic molecules. The large basis set dependence shows that an estimation of the interaction energy at the basis set limit is necessary for a quantitative evaluation of the interaction energies in the complexes.

Calculated MP2 and CCSD(T) interaction energies for the complexes summarized in Table 2S show that the MP2 method overestimates the attraction considerably compared with the more reliable CCSC(T) method as in the cases of other aromatic complexes. The comparison shows that electron correlation effects beyond MP2 are significant and that a CCSD(T) level correction is necessary.

\section{Estimation of MP2 interaction energy at the basis set limit}

The MP2 interaction energy at the basis set limit $\left[\mathrm{E}_{\mathrm{MP} 2 \text { (limit) }}\right.$ was estimated by Helgaker's method from the calculated EMP2 using the aug-cc-pVDZ and aug-cc-pVTZ basis sets as summarized in Table 3S. In Helgaker's method the calculated EMP2 values were fitted to a form $\mathrm{a}+\mathrm{b} \mathrm{X}^{-3}$ (where $\mathrm{X}$ is 2 for aug-cc-pVDZ and 3 for aug-cc-pVTZ). The EMP2(limit) value was then estimated by an extrapolation. Helgaker's method was originally proposed for an estimation of the electron correlation contribution at the basis set limit.

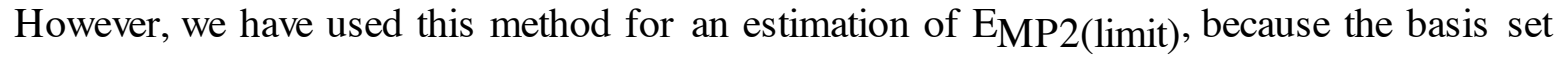
dependence of $\mathrm{E}_{\mathrm{HF}}$ is small.

\section{Estimation procedure of CCSD(T) interaction energy at the basis set limit}

The strong basis set dependence and substantial electron correlation effects beyond MP2 show that an estimation of the $\operatorname{CCSD}(\mathrm{T})$ interaction energy at the basis set limit $\left[\mathrm{E}_{\mathrm{CCSD}}(\mathrm{T})(\right.$ limit)$]$ is necessary for a quantitative analysis of interaction energies in the 
complexes. ECCSD(T)(limit) value (Table 4S) was estimated with ARS-E model in this work. In this model the $\mathrm{ECCSD}(\mathrm{T})($ limit) was estimated according to equation (1)

$\mathrm{ECCSD}(\mathrm{T})($ limit $)=\mathrm{EMP} 2($ limit $)+\Delta \mathrm{CCSD}(\mathrm{T})($ limit $)$,

where $\Delta \operatorname{CCSD}(\mathrm{T})($ limit $)$ denotes the $\operatorname{CCSD}(\mathrm{T})$ correction term $[\Delta \operatorname{CCSD}(\mathrm{T})=\operatorname{ECCSD}(\mathrm{T})$ EMP2] at the basis set limit. EMP2 and $\operatorname{ECCSD(T)~denote~MP2~and~} \operatorname{CCSD}(\mathrm{T})$ interaction energies. EMP2(limit) denotes the EMP2 at the basis set limit estimated by Helgaker's method.

The $\Delta \operatorname{CCSD}(\mathrm{T})$ (limit) was estimated by equation (2)

$\Delta \operatorname{CCSD}(\mathrm{T})($ limit $)=\Delta \operatorname{CCSD}(\mathrm{T})(\mathrm{M})+\Delta(\mathrm{M}) \Delta \operatorname{CCSD}(\mathrm{T})$,

where $\Delta \operatorname{CCSD}(\mathrm{T})(\mathrm{M})$ denotes $\triangle \mathrm{CCSD}(\mathrm{T})$ obtained using a Medium size basis set. The $\Delta \mathrm{CCSD}(\mathrm{T})$ has a weak basis set dependence. $\Delta(\mathrm{M}) \Delta \mathrm{CCSD}(\mathrm{T})$ is a correction term for slight underestimation of the $\Delta \operatorname{CCSD}(\mathrm{T})$ by the Medium size basis set. The $\Delta(\mathrm{M}) \Delta \operatorname{CCSD}(\mathrm{T})$ corresponds to a difference between the $\Delta \operatorname{CCSD}(\mathrm{T})$ at the basis set limit $[\Delta \operatorname{CCSD}(\mathrm{T})($ limit $)]$ and the $\Delta \operatorname{CCSD}(\mathrm{T})(\mathrm{M})$. The 6-31G* basis set was used for the Medium size basis set in this work.

The $\Delta(\mathrm{M}) \Delta \mathrm{CCSD}(\mathrm{T})$ was estimated according to equation (3)

$$
\begin{aligned}
& \Delta(\mathrm{M}) \Delta \mathrm{CCSD}(\mathrm{T})=\mathrm{F}_{\Delta \mathrm{CCSD}}(\mathrm{T}) \times \Delta(\mathrm{M}) \mathrm{E}_{\operatorname{corr}(\mathrm{MP} 2)} \\
& =\mathrm{F}_{\Delta} \mathrm{CCSD}(\mathrm{T}) \times\left[\mathrm{E}_{\operatorname{corr}(\mathrm{MP} 2)(\text { limit })}-\mathrm{E}_{\operatorname{corr}(\mathrm{MP} 2)(\mathrm{M})]},\right.
\end{aligned}
$$


where $\mathrm{E}_{\text {corr(MP2)(M) denotes }} \mathrm{E}_{\text {corr(MP2) }}\left(=\mathrm{E}_{\mathrm{MP}}\right.$ - $\left.-\mathrm{E}_{\mathrm{HF}}\right)$ obtained using the Medium size basis set. $\Delta(\mathrm{M}) \mathrm{E}_{\text {corr }}(\mathrm{MP} 2)\left[=\mathrm{E}_{\mathrm{corr}}(\mathrm{MP} 2)(\right.$ limit $\left.)-\mathrm{E}_{\mathrm{corr}}(\mathrm{MP} 2)(\mathrm{M})\right]$ is underestimation of the $\mathrm{E}_{\text {corr(MP2) }}$ by the Medium size basis set. $\mathrm{F}_{\Delta \mathrm{CCSD}}(\mathrm{T})$ is a parameter used for estimation of the $\Delta(\mathrm{M}) \Delta \operatorname{CCSD}(\mathrm{T})$. Calculated $\operatorname{ECCSD}(\mathrm{T})$ 's for the benzene-pyridine complexes (Table $2 \mathrm{~S}$ ) show that the $\mathrm{E}_{\mathrm{corr}}\left(\mathrm{CCSD}(\mathrm{T})\right.$ ) is always $75 \pm 5 \%$ of the $\mathrm{E}_{\mathrm{corr}}(\mathrm{MP} 2)$. Similar results were reported in $\operatorname{CCSD}(\mathrm{T})$ calculations for the benzene, naphthalene, toluene and thiophene dimers. The $\mathrm{E}_{\operatorname{corr}}(\operatorname{CCSD}(\mathrm{T}))$ for the benzene complexes with pyridinium and $N$-methylpyridinium are $75 \pm 10 \%$ of the $\mathrm{E}_{\mathrm{cor}}(\mathrm{MP} 2)$. These results suggest that we can assume that the $\Delta(\mathrm{M}) \Delta \mathrm{CCSD}(\mathrm{T})$ is approximately $25 \pm 10 \%$ of the absolute value of $\Delta(\mathrm{M}) \mathrm{E}_{\mathrm{corr}}(\mathrm{MP} 2)$. Therefore $\mathrm{F} \Delta \mathrm{CCSD}(\mathrm{T})=-0.25$ was used for the estimation of $\Delta(\mathrm{M}) \Delta \mathrm{CCSD}(\mathrm{T})$ using equation (3) in this work.

\section{Basis set effects on calculated atomic charges}

Atomic charges of benzene, pyridine, pyridinium and $N$-methylpyridinium were obtained by electrostatic potential fitting using the Merz-Singh-Kollman scheme from MP2 level wave functions using several basis sets as summarized in Table $5 \mathrm{~S}$. The basis set effects on the calculated atomic charge distributions are small, if the basis sets including polarization functions $\left(6-31 G^{*}\right.$ or larger) are used. The atomic charges obtained using the MP2/cc-pVTZ level wave functions are very close to those obtained using the very large cc-pVQZ basis set.

\section{Complete Ref. 59}

(59) Gaussian 03, Revision D.01, Frisch, M. J.; Trucks, G. W.; Schlegel, H. B.; Scuseria, G. E.; Robb, M. A.; Cheeseman, J. R.; Montgomery, Jr., J. A.; Vreven, T.; Kudin, K. N.; Burant, J. C.; Millam, J. M.; Iyengar, S. S.; Tomasi, J.; Barone, V.; Mennucci, B.; Cossi, M.; Scalmani, G.; Rega, N.; Petersson, G. A.; Nakatsuji, H.; Hada, M.; Ehara, M.; Toyota, K.; Fukuda, R.; Hasegawa, J.; Ishida, M.; Nakajima, T.; Honda, Y.; Kitao, O.; Nakai, H.; Klene, M.; Li, X.; 
Knox, J. E.; Hratchian, H. P.; Cross, J. B.; Bakken, V.; Adamo, C.; Jaramillo, J.; Gomperts, R.; Stratmann, R. E.; Yazyev, O.; Austin, A. J.; Cammi, R.; Pomelli, C.; Ochterski, J. W.; Ayala, P. Y.; Morokuma, K.; Voth, G. A.; Salvador, P.; Dannenberg, J. J.; Zakrzewski, V. G.; Dapprich, S.; Daniels, A. D.; Strain, M. C.; Farkas, O.; Malick, D. K.; Rabuck, A. D.; Raghavachari, K.; Foresman, J. B.; Ortiz, J. V.; Cui, Q.; Baboul, A. G.; Clifford, S.; Cioslowski, J.; Stefanov, B. B.; Liu, G.; Liashenko, A.; Piskorz, P.; Komaromi, I.; Martin, R. L.; Fox, D. J.; Keith, T.; Al-Laham, M. A.; Peng, C. Y.; Nanayakkara, A.; Challacombe, M.; Gill, P. M. W.; Johnson, B.; Chen, W.; Wong, M. W.; Gonzalez, C.; and Pople, J. A.; Gaussian, Inc., Wallingford CT, 2004. 
Table 1S. Calculated HF and MP2 Interaction Energies for Benzene Complexes with Pyridine, Pyridinium and $N$-Methylpyridinium ${ }^{\mathrm{a}}$

\begin{tabular}{|c|c|c|c|c|}
\hline & $b f b$ & $\mathrm{E}_{\mathrm{HF}}{ }^{\mathrm{c}}$ & $\mathrm{E}_{\mathrm{MP} 2}{ }^{\mathrm{c}}$ & $\mathrm{E}_{\mathrm{MP} 2 \operatorname{corr}^{\mathrm{d}}}$ \\
\hline$\frac{\text { Basis set }}{\text { Benzene-pyridine 1f }}$ & & & & \\
\hline $6-31 G^{*}$ & 202 & 3.86 & -1.22 & -5.08 \\
\hline $6-311 \mathrm{G}^{* *}$ & 282 & 3.50 & -2.84 & -6.34 \\
\hline cc-pVDZ & 223 & 3.56 & -2.42 & -5.98 \\
\hline cc-pVTZ & 514 & 3.49 & -4.26 & -7.75 \\
\hline cc-pVQZ & 990 & 3.47 & -4.87 & -8.34 \\
\hline aug-cc-pVDZ & 375 & 3.46 & -4.74 & -8.20 \\
\hline aug-cc-pVTZ & 805 & 3.45 & -5.10 & -8.54 \\
\hline basis set limit & & $3.45^{\mathrm{e}}$ & $-5.25^{f}$ & $-8.70 \mathrm{~g}$ \\
\hline \multicolumn{5}{|c|}{ Benzene-pyridinium $\mathbf{2 g}$} \\
\hline $6-31 G^{*}$ & 204 & -9.32 & -13.82 & -4.50 \\
\hline $6-311 \mathrm{G} * *$ & 288 & -9.10 & -14.42 & -5.32 \\
\hline cc-pVDZ & 228 & -8.78 & -13.82 & -5.04 \\
\hline cc-pVTZ & 528 & -9.24 & -16.22 & -6.98 \\
\hline cc-pVQZ & 1020 & -9.21 & -16.83 & -7.62 \\
\hline aug-cc-pVDZ & 384 & -9.15 & -15.91 & -6.76 \\
\hline aug-cc-pVTZ & 828 & -9.13 & -16.79 & -7.66 \\
\hline basis set limit & & $-9.13^{\mathrm{e}}$ & $-17.17^{f}$ & $-8.04 \mathrm{~g}$ \\
\hline \multicolumn{5}{|c|}{ Benzene- $N$-methylpyridinium $\mathbf{3 f}$} \\
\hline $6-31 \mathrm{G}^{*}$ & 223 & -1.78 & -7.54 & -5.76 \\
\hline $6-311 \mathrm{G}^{* *}$ & 318 & -1.97 & -9.27 & -7.30 \\
\hline cc-pVDZ & 252 & -1.75 & -8.57 & -6.81 \\
\hline cc-pVTZ & 586 & -1.82 & -10.88 & -9.05 \\
\hline cc-pVQZ & 1135 & -1.83 & -11.57 & -9.73 \\
\hline aug-cc-pVDZ & 425 & -1.80 & -11.17 & -9.37 \\
\hline aug-cc-pVTZ & 920 & -1.78 & -11.70 & -9.91 \\
\hline basis set limit & & $-1.78^{\mathrm{e}}$ & $-11.92^{f}$ & $-10.14 \mathrm{~g}$ \\
\hline
\end{tabular}

${ }^{a}$ Geometries of the complexes are shown in Figs. 5,7 and 9. Energies in kcal/mol.

$\mathrm{b}$ Number of basis functions used for the calculation of the complex.

c BSSE corrected interaction energies.

$\mathrm{d}$ MP2 correlation interaction energies $\left(=\mathrm{E}_{\mathrm{MP} 2}-\mathrm{E}_{\mathrm{HF}}\right)$. 
e HF/aug-cc-pVTZ interaction energies were used as the HF level interaction energies at the basis set limit $\left[\mathrm{E}_{\mathrm{HF}}(\mathrm{limit})\right]$

f Estimated MP2 level interaction energies at the basis set limit [E $\mathrm{EP}$ 2(limit)] by Helgaker's method.

$\mathrm{g}$ Estimated MP2 correlation interaction energies at the basis set limit $\left[\mathrm{E}_{\mathrm{corr}}(\mathrm{MP} 2)(\mathrm{limit})=\right.$ $\mathrm{E}_{\mathrm{MP} 2(\text { limit })}-\mathrm{E}_{\mathrm{HF}}$ (limit)]. 
Table 2S. Calculated MP2 and CCSD(T) Interaction Energies for Benzene Complexes with Pyridine, Pyridinium and $N$-Methylpyridinium Using 6-31G* Basis Set ${ }^{\mathrm{a}}$

\begin{tabular}{|c|c|c|c|c|c|c|}
\hline basis set, geometry & $\mathrm{E}_{\mathrm{HF}}{ }^{\mathrm{b}}$ & $\mathrm{E}_{\mathrm{MP} 2}{ }^{\mathrm{b}}$ & $\mathrm{E}_{\mathrm{CCSD}(\mathrm{T})}{ }^{\mathrm{b}}$ & $\mathrm{E}_{\operatorname{corr}(\mathrm{MP} 2)}{ }^{\mathrm{c}}$ & $\mathrm{E}_{\operatorname{corr}(\operatorname{CSSD}(\mathrm{T}))^{\mathrm{d}}}$ & $\begin{array}{c}\mathrm{E}_{\mathrm{corr}(\mathrm{CCSD}(\mathrm{T})) /} \\
\mathrm{E}_{\mathrm{corr}(\mathrm{MP} 2)}\end{array}$ \\
\hline \multicolumn{7}{|l|}{ Benzene-pyridine } \\
\hline 1f & 3.86 & -1.22 & 0.09 & -5.08 & -3.77 & 0.74 \\
\hline $1 g$ & 4.00 & -0.52 & 0.54 & -4.51 & -3.46 & 0.77 \\
\hline $1 \mathrm{~h}$ & 0.26 & -2.08 & -1.49 & -2.33 & -1.74 & 0.75 \\
\hline $1 \mathbf{i}$ & -0.06 & -1.98 & -1.48 & -1.92 & -1.42 & 0.74 \\
\hline $\mathbf{1 j}$ & 0.20 & -1.75 & -1.30 & -1.95 & -1.50 & 0.77 \\
\hline \multicolumn{7}{|c|}{ Benzene-pyridinium } \\
\hline $2 f$ & -0.38 & -6.78 & -4.85 & -6.40 & -4.46 & 0.70 \\
\hline $2 \mathrm{~g}$ & -9.32 & -13.82 & -12.30 & -4.50 & -2.98 & 0.66 \\
\hline $2 h$ & -4.64 & -7.15 & -6.28 & -2.51 & -1.63 & 0.65 \\
\hline $2 \mathbf{i}$ & 0.69 & -1.67 & -1.31 & -2.36 & -2.00 & 0.85 \\
\hline \multicolumn{7}{|c|}{ Benzene- $N$-methylpyridinium } \\
\hline $3 f$ & -1.78 & -7.54 & -6.08 & -5.76 & -4.30 & 0.75 \\
\hline $3 g$ & 0.16 & -6.37 & -4.43 & -6.53 & -4.60 & 0.70 \\
\hline $3 h$ & -4.51 & -7.58 & -6.66 & -3.07 & -2.15 & 0.70 \\
\hline $3 \mathbf{i}$ & -4.27 & -6.75 & -5.91 & -2.47 & -1.63 & 0.66 \\
\hline 3j & -3.52 & -5.78 & -5.24 & -2.25 & -1.72 & 0.76 \\
\hline 3k & 0.93 & -1.69 & -1.29 & -2.62 & -2.22 & 0.85 \\
\hline
\end{tabular}

a Geometries of the complexes are shown in Figs. 5, 7 and 9. Energies in kcal/mol.

b BSSE corrected interaction energies.

${ }^{\mathrm{c}} \mathrm{MP} 2$ correlation interaction energies [= $\left.\mathrm{E}_{\mathrm{MP} 2}-\mathrm{E}_{\mathrm{HF}}\right]$.

$\mathrm{d} \operatorname{CCSD}(\mathrm{T})$ correlation interaction energies $\left[=\mathrm{E}_{\mathrm{CCSD}}(\mathrm{T})-\mathrm{E}_{\mathrm{HF}}\right]$. 
Table 3S. MP2 Interaction Energies and Estimated MP2 Interaction Energies at the Basis Set Limit for Benzene Complexes with Pyridine, Pyridinium and $N$-Methylpyridiniuma

\begin{tabular}{llcc}
\hline & aug-cc-pVDZb & aug-cc-pVTZb & $\mathrm{E}_{\mathrm{MP} 2 \text { (limit) }}$ \\
\hline Benzene-pyridine & & & \\
$\mathbf{1 f}$ & -4.74 & -5.10 & -5.25 \\
$\mathbf{1 g}$ & -3.64 & -3.94 & -4.06 \\
$\mathbf{1 h}$ & -3.62 & -3.88 & -3.99 \\
$\mathbf{1 i}$ & -3.41 & -3.68 & -3.79 \\
$\mathbf{1 j}$ & -3.17 & -3.37 & -3.45 \\
Benzene-pyridinium & & & \\
$\mathbf{2 f}$ & -10.60 & -11.13 & -11.35 \\
$\mathbf{2 g}$ & -15.91 & -16.79 & -17.17 \\
$\mathbf{2 h}$ & -9.04 & -9.60 & -9.83 \\
$\mathbf{2 i}$ & -3.26 & -3.46 & -3.54 \\
Benzene- $N$-methylpyridinium & & \\
$\mathbf{3 f}$ & -11.17 & -11.70 & -11.92 \\
$\mathbf{3 g}$ & -10.19 & -10.70 & -10.91 \\
$\mathbf{3 h}$ & -8.91 & -9.34 & -9.53 \\
$\mathbf{3 i}$ & -8.55 & -9.07 & -9.29 \\
$\mathbf{3 j}$ & -7.26 & -7.57 & -7.71 \\
$\mathbf{3 k}$ & -3.39 & -3.62 & -3.72
\end{tabular}

a Energies in kcal/mol. Geometries of the complexes are shown in Figs. 5, 7 and 9.

b BSSE corrected MP2 interaction energies (EMP2).

c Estimated MP2 interaction energies at the basis set limit by Helgaker's method. See text. 
Table 4S. Estimated CCSD(T) Interaction Energies at the Basis Set Limit by ARS-E Model Using 6-31G* Basis Set as Medium-Size Basis Set for Benzene Complexes with Pyridine, Pyridinium and $N$-Methylpyridiniuma

\begin{tabular}{|c|c|c|c|c|c|c|}
\hline Dimer & $\mathrm{E}_{\mathrm{HF} \text { (limit) }} \mathrm{b}$ & $\mathrm{E}_{\mathrm{MP} 2(\text { limit }}{ }^{\mathrm{c}}$ & $\mathrm{E}_{\text {corr(MP2)(limit) }}{ }^{\mathrm{d}}$ & $\mathrm{E}_{\mathrm{HF}(\mathrm{M})}{ }^{\mathrm{e}}$ & $\mathrm{E}_{\mathrm{MP} 2(\mathrm{M})}{ }^{\mathrm{e}}$ & $\mathrm{E}_{\mathrm{CCSD}(\mathrm{T})(\mathrm{M})}{ }^{\mathrm{e}}$ \\
\hline \multicolumn{7}{|c|}{ Benzene-pyridine } \\
\hline 1f & 3.45 & -5.25 & -8.69 & 3.86 & -1.22 & 0.09 \\
\hline $1 g$ & 3.63 & -4.06 & -7.69 & 4.00 & -0.52 & 0.54 \\
\hline $1 \mathrm{~h}$ & 0.44 & -3.99 & -4.43 & 0.26 & -2.08 & -1.49 \\
\hline $\mathbf{1 i}$ & 0.05 & -3.79 & -3.84 & -0.06 & -1.98 & -1.48 \\
\hline $\mathbf{1 j}$ & 0.23 & -3.45 & -3.68 & 0.20 & -1.75 & -1.30 \\
\hline \multicolumn{7}{|c|}{ Benzene-pyridinium } \\
\hline $2 f$ & -0.63 & -11.35 & -10.72 & -0.38 & -6.78 & -4.85 \\
\hline $2 g$ & -9.13 & -17.17 & -8.03 & -9.32 & -13.82 & -12.30 \\
\hline $2 \mathrm{~h}$ & -4.76 & -9.83 & -5.07 & -4.64 & -7.15 & -6.28 \\
\hline $2 \mathbf{i}$ & 0.60 & -3.54 & -4.15 & 0.69 & -1.67 & -1.31 \\
\hline \multicolumn{7}{|c|}{ Benzene- $N$-methylpyridinium } \\
\hline $3 f$ & -1.78 & -11.92 & -10.13 & -1.78 & -7.54 & -6.08 \\
\hline $3 g$ & -0.02 & -10.91 & -10.89 & 0.16 & -6.37 & -4.43 \\
\hline $3 h$ & -4.01 & -9.53 & -5.52 & -4.51 & -7.58 & -6.66 \\
\hline $3 \mathbf{i}$ & -4.32 & -9.29 & -4.98 & -4.27 & -6.75 & -5.91 \\
\hline 3j & -3.36 & -7.71 & -4.35 & -3.52 & -5.78 & -5.24 \\
\hline 3k & 0.88 & -3.72 & -4.59 & 0.93 & -1.69 & -1.29 \\
\hline \multicolumn{3}{|c|}{$\begin{array}{l}\operatorname{CCSD}(\mathrm{T})(\mathrm{M})^{\mathrm{f}} \mathrm{E}_{\operatorname{corr}(\mathrm{MP} 2)(\mathrm{M})^{\mathrm{g}}} \\
\text { Dimer }\end{array}$} & $\Delta(\mathrm{M}) \mathrm{E}_{\text {corr(}(\mathrm{MP} 2)}{ }^{\mathrm{h}}$ & \multicolumn{3}{|c|}{$\Delta(\mathrm{M}) \Delta \operatorname{CCSD}(\mathrm{T}){ }^{\mathrm{i} \Delta \operatorname{CCSD}(\mathrm{T})}(\text { (limit })^{\mathrm{j}} \mathrm{E}_{\operatorname{CCSD}(\mathrm{T})(\text { limit })}{ }^{\mathrm{k}}$} \\
\hline \multicolumn{7}{|c|}{ Benzene-pyridine } \\
\hline 1f & 1.31 & -5.08 & 3.62 & 0.90 & 2.21 & -3.04 \\
\hline $1 g$ & 1.05 & -4.51 & 3.18 & 0.79 & 1.85 & -2.22 \\
\hline $1 \mathrm{~h}$ & 0.59 & -2.33 & 2.10 & 0.52 & 1.12 & -2.87 \\
\hline $1 \mathbf{i}$ & 0.50 & -1.92 & 1.92 & 0.48 & 0.97 & -2.81 \\
\hline $\mathbf{1 j}$ & 0.45 & -1.95 & 1.74 & 0.43 & 0.88 & -2.57 \\
\hline \multicolumn{7}{|c|}{ Benzene-pyridinium } \\
\hline $2 f$ & 1.93 & -6.40 & 4.32 & 1.08 & 3.01 & -8.34 \\
\hline $2 \mathrm{~g}$ & 1.52 & -4.50 & 3.53 & 0.88 & 2.40 & -14.77 \\
\hline $2 \mathrm{~h}$ & 0.88 & -2.51 & 2.56 & 0.64 & 1.51 & -8.31 \\
\hline $2 \mathbf{i}$ & 0.36 & -2.36 & 1.79 & 0.45 & 0.81 & -2.74 \\
\hline \multicolumn{7}{|c|}{ Benzene- $N$-methylpyridinium } \\
\hline 3f & 1.46 & -5.76 & 4.38 & 1.09 & 2.55 & -9.36 \\
\hline $3 g$ & 1.94 & -6.53 & 4.35 & 1.09 & 3.03 & -7.88 \\
\hline $3 h$ & 0.92 & -3.07 & 2.45 & 0.61 & 1.53 & -8.00 \\
\hline $3 \mathbf{i}$ & 0.84 & -2.47 & 2.51 & 0.63 & 1.46 & -7.83 \\
\hline 3j & 0.54 & -2.25 & 2.10 & 0.52 & 1.06 & -6.65 \\
\hline $3 k$ & 0.40 & -2.62 & 1.97 & 0.49 & 0.90 & -2.82 \\
\hline
\end{tabular}


a Geometries of the complexes are shown in Figs. 5, 7 and 9. Energies in kcal/mol.

b HF interaction energies at the basis set limit. HF/aug-cc-pVTZ interaction energies were used.

c Estimated MP2 interaction energies at the basis set limit by Helgaker's method. See text.

d MP2 correlation interaction energies at the basis set limit [= EMP2(limit) $\left.-E_{H F(l i m i t)}\right]$. See text.

e HF, MP2 and CCSD(T) interaction energies calculated using the Medium size basis sets. See text.

${ }^{\mathrm{f}} \mathrm{CCSD}(\mathrm{T})$ correction terms calculated using the Medium size basis set $[=\operatorname{ECCSD}(\mathrm{T})(\mathrm{M})$ EMP2(M)]. See text.

g MP2 correlation interaction energies calculated using the Medium size basis set [= EMP2(M) - EHF(M)]. See text.

$\mathrm{h}$ Underestimation of MP2 correlation interaction energies by the Medium size basis set [= $\mathrm{E}_{\text {corr(MP2)(limit) }}-\mathrm{E}_{\text {corr(MP2)(M) }}$. See text.

${ }^{\mathrm{i}}$ Estimated underestimation of $\operatorname{CCSD}(\mathrm{T})$ correction terms by the Medium size basis set [= $0.25 \times \Delta(\mathrm{M}) \mathrm{E}_{\mathrm{corr}(\mathrm{MP} 2)]}$. See text.

j Estimated $\operatorname{CCSD}(\mathrm{T})$ correction terms at the basis set limit $[=\Delta \operatorname{CCSD}(\mathrm{T})(\mathrm{M})+$ $\Delta(\mathrm{M}) \Delta \mathrm{CCSD}(\mathrm{T})]$. See text.

$\mathrm{k}$ Estimated $\operatorname{CCSD}(\mathrm{T})$ interaction energies at the basis set limit $\left[=\mathrm{E}_{\mathrm{MP}}\right.$ (limit) + $\Delta \operatorname{CCSD}(\mathrm{T})($ limit)]. See text. 
Table 5S. Atomic Charges of Benzene, Pyridine, Pyridinium and $N$-Methylpyridinium Obtained by Electrostatic Potential Fitting Using the Merz-Singh-Kollman Scheme from MP2 Level Wave Functions Using Several Basis Sets ${ }^{\mathrm{a}}$

\begin{tabular}{|c|c|c|c|c|c|c|c|}
\hline & $6-31 G$ & $6-31 G^{*}$ & $6-311 G^{*}$ & $6-311 \mathrm{G}^{* *}$ & cc-pVDZ & cc-pVTZ & cc-pVQZ \\
\hline \multicolumn{8}{|c|}{ Benzene } \\
\hline $\mathrm{C} 1$ & -0.12 & -0.13 & -0.14 & -0.13 & -0.12 & -0.13 & -0.13 \\
\hline $\mathrm{C} 2$ & -0.12 & -0.13 & -0.14 & -0.13 & -0.12 & -0.13 & -0.13 \\
\hline $\mathrm{C} 3$ & -0.12 & -0.13 & -0.14 & -0.13 & -0.12 & -0.13 & -0.13 \\
\hline $\mathrm{C} 4$ & -0.12 & -0.13 & -0.14 & -0.13 & -0.12 & -0.13 & -0.13 \\
\hline $\mathrm{C} 5$ & -0.11 & -0.12 & -0.13 & -0.12 & -0.12 & -0.12 & -0.13 \\
\hline C6 & -0.11 & -0.12 & -0.13 & -0.12 & -0.12 & -0.12 & -0.13 \\
\hline $\mathrm{H} 7$ & 0.12 & 0.13 & 0.14 & 0.13 & 0.12 & 0.13 & 0.13 \\
\hline H8 & 0.12 & 0.13 & 0.14 & 0.13 & 0.12 & 0.13 & 0.13 \\
\hline H9 & 0.12 & 0.13 & 0.14 & 0.13 & 0.12 & 0.13 & 0.13 \\
\hline H10 & 0.12 & 0.13 & 0.14 & 0.13 & 0.12 & 0.13 & 0.13 \\
\hline H11 & 0.12 & 0.12 & 0.14 & 0.13 & 0.12 & 0.13 & 0.13 \\
\hline H12 & 0.12 & 0.12 & 0.14 & 0.13 & 0.12 & 0.13 & 0.13 \\
\hline \multicolumn{8}{|c|}{ Pyridine } \\
\hline $\mathrm{C} 1$ & 0.31 & 0.23 & 0.23 & 0.24 & 0.22 & 0.24 & 0.26 \\
\hline $\mathrm{C} 2$ & -0.54 & -0.48 & -0.51 & -0.49 & -0.46 & -0.49 & -0.51 \\
\hline $\mathrm{C} 3$ & 0.59 & 0.46 & 0.46 & 0.47 & 0.43 & 0.46 & 0.47 \\
\hline $\mathrm{C} 4$ & -0.54 & -0.48 & -0.51 & -0.49 & -0.46 & -0.49 & -0.51 \\
\hline $\mathrm{C} 5$ & 0.59 & 0.46 & 0.46 & 0.47 & 0.43 & 0.46 & 0.47 \\
\hline N6 & -0.76 & -0.64 & -0.63 & -0.63 & -0.59 & -0.63 & -0.65 \\
\hline $\mathrm{H} 7$ & 0.05 & 0.07 & 0.08 & 0.07 & 0.07 & 0.07 & 0.07 \\
\hline H8 & 0.18 & 0.18 & 0.19 & 0.18 & 0.17 & 0.18 & 0.18 \\
\hline $\mathrm{H} 9$ & -0.02 & 0.01 & 0.02 & 0.01 & 0.01 & 0.02 & 0.01 \\
\hline H10 & 0.18 & 0.18 & 0.19 & 0.18 & 0.17 & 0.18 & 0.18 \\
\hline H11 & -0.02 & 0.01 & 0.02 & 0.01 & 0.01 & 0.02 & 0.01 \\
\hline \multicolumn{8}{|c|}{ Pyridinium } \\
\hline $\mathrm{C} 1$ & 0.01 & -0.01 & -0.02 & -0.01 & 0.00 & -0.01 & 0.00 \\
\hline $\mathrm{C} 2$ & -0.05 & -0.06 & -0.07 & -0.06 & -0.06 & -0.06 & -0.06 \\
\hline $\mathrm{C} 3$ & 0.00 & -0.01 & -0.04 & -0.02 & -0.02 & -0.02 & -0.01 \\
\hline $\mathrm{C} 4$ & -0.05 & -0.06 & -0.07 & -0.06 & -0.06 & -0.06 & -0.06 \\
\hline $\mathrm{C} 5$ & 0.00 & -0.01 & -0.04 & -0.02 & -0.02 & -0.02 & -0.01 \\
\hline N6 & -0.07 & -0.09 & -0.05 & -0.04 & -0.03 & -0.06 & -0.07 \\
\hline $\mathrm{H} 7$ & 0.15 & 0.16 & 0.17 & 0.16 & 0.16 & 0.16 & 0.16 \\
\hline H8 & 0.17 & 0.18 & 0.19 & 0.18 & 0.17 & 0.18 & 0.18 \\
\hline H9 & 0.18 & 0.19 & 0.20 & 0.18 & 0.18 & 0.18 & 0.18 \\
\hline H10 & 0.17 & 0.18 & 0.19 & 0.18 & 0.17 & 0.18 & 0.18 \\
\hline H11 & 0.18 & 0.19 & 0.20 & 0.18 & 0.18 & 0.18 & 0.18 \\
\hline H12 & 0.34 & 0.35 & 0.34 & 0.33 & 0.32 & 0.33 & 0.33 \\
\hline
\end{tabular}




$\begin{array}{lccccccc}\text { C1 } & 0.01 & -0.02 & -0.03 & -0.02 & -0.02 & -0.02 & -0.02 \\ \text { C2 } & -0.09 & -0.09 & -0.10 & -0.08 & -0.08 & -0.08 & -0.08 \\ \text { C3 } & -0.04 & -0.07 & -0.10 & -0.08 & -0.08 & -0.08 & -0.08 \\ \text { C4 } & -0.09 & -0.09 & -0.10 & -0.08 & -0.08 & -0.08 & -0.08 \\ \text { C5 } & -0.04 & -0.07 & -0.10 & -0.08 & -0.08 & -0.08 & -0.08 \\ \text { N6 } & 0.24 & 0.27 & 0.33 & 0.30 & 0.28 & 0.28 & 0.28 \\ \text { H7 } & 0.15 & 0.16 & 0.18 & 0.16 & 0.16 & 0.17 & 0.17 \\ \text { H8 } & 0.18 & 0.19 & 0.20 & 0.18 & 0.18 & 0.19 & 0.19 \\ \text { H9 } & 0.17 & 0.19 & 0.19 & 0.18 & 0.18 & 0.18 & 0.18 \\ \text { H10 } & 0.18 & 0.19 & 0.20 & 0.18 & 0.18 & 0.19 & 0.19 \\ \text { H11 } & 0.17 & 0.19 & 0.19 & 0.18 & 0.18 & 0.18 & 0.18 \\ \text { C12 } & -0.39 & -0.44 & -0.50 & -0.44 & -0.39 & -0.43 & -0.44 \\ \text { H13 } & 0.19 & 0.20 & 0.21 & 0.20 & 0.19 & 0.20 & 0.20 \\ \text { H14 } & 0.19 & 0.20 & 0.21 & 0.20 & 0.18 & 0.20 & 0.20 \\ \text { H15 } & 0.19 & 0.20 & 0.21 & 0.20 & 0.18 & 0.20 & 0.20\end{array}$

a The MP2/6-311G** level optimized geometries were used for the calculations. Numbering of atoms is shown in Fig.1S. 

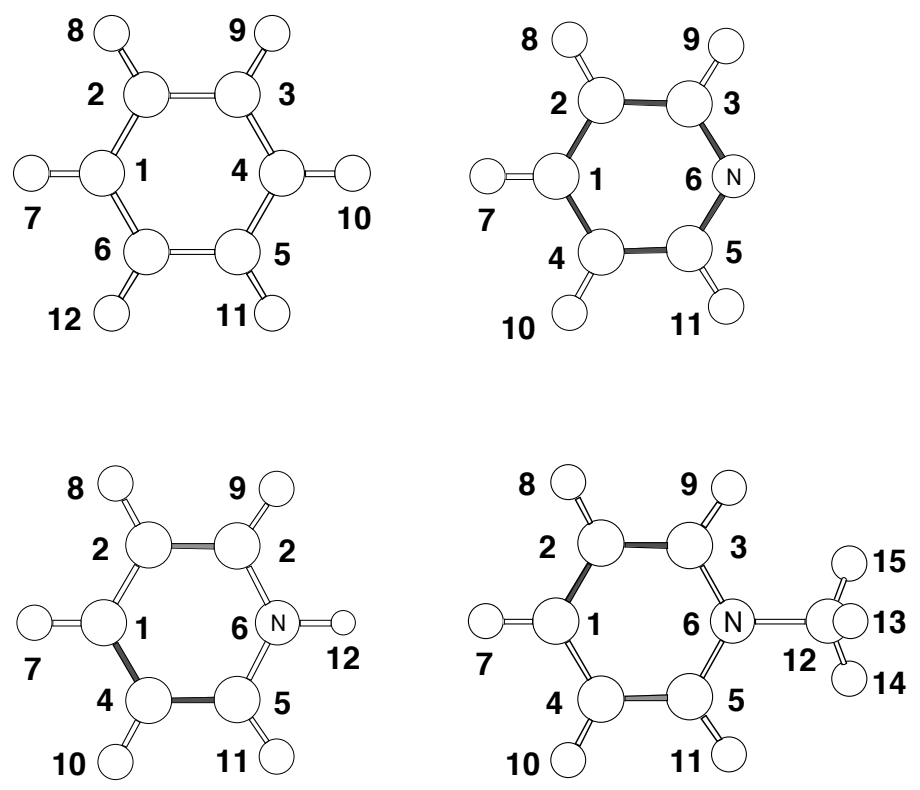

Figure 1S Numbering of atoms in Table 1S. 


\begin{tabular}{|c|c|c|c|c|c|}
\hline \multirow{2}{*}{$\begin{array}{l}\text { Center } \\
\text { Number }\end{array}$} & Atomic & \multicolumn{2}{|c|}{ Atomic } & \multicolumn{2}{|c|}{ Coordinates (Angstroms) } \\
\hline & Numbe & & Type & $\mathrm{X}$ & Z \\
\hline 1 & 6 & 0 & 1.900000 & 1.390040 & 0.000000 \\
\hline 2 & 6 & 0 & 1.900000 & 0.690480 & 1.211673 \\
\hline 3 & 6 & 0 & 1.900000 & -0.708640 & 1.211673 \\
\hline 4 & 6 & 0 & 1.900000 & -1.408200 & 0.000000 \\
\hline 5 & 6 & 0 & 1.900000 & -0.708640 & -1.211673 \\
\hline 6 & 6 & 0 & 1.900000 & 0.690480 & -1.211673 \\
\hline 7 & 1 & 0 & 1.900000 & 2.476447 & 0.000000 \\
\hline 8 & 1 & 0 & 1.900000 & 1.233683 & 2.152530 \\
\hline 9 & 1 & 0 & 1.900000 & -1.251844 & 2.152530 \\
\hline 10 & 1 & 0 & 1.900000 & -2.494607 & 0.000000 \\
\hline 11 & 1 & 0 & 1.900000 & -1.251844 & -2.152530 \\
\hline 12 & 1 & 0 & 1.900000 & 1.233683 & -2.152530 \\
\hline 13 & 6 & 0 & -1.900000 & 1.401020 & 0.000000 \\
\hline 14 & 6 & 0 & -1.900000 & 0.683530 & 1.197974 \\
\hline 15 & 6 & 0 & -1.900000 & -0.713605 & 1.143425 \\
\hline 16 & 6 & 0 & -1.900000 & 0.683530 & -1.197974 \\
\hline 17 & 6 & 0 & -1.900000 & -0.713605 & -1.143425 \\
\hline 18 & 7 & 0 & -1.900000 & -1.419180 & 0.000000 \\
\hline 19 & 1 & 0 & -1.900000 & 2.487120 & 0.000000 \\
\hline 20 & 1 & 0 & -1.900000 & 1.190647 & 2.157849 \\
\hline 21 & 1 & 0 & -1.900000 & -1.299005 & 2.060513 \\
\hline 22 & 1 & 0 & -1.900000 & 1.190647 & -2.157849 \\
\hline 23 & 1 & 0 & -1.900000 & -1.299005 & -2.060513 \\
\hline
\end{tabular}

$\mathrm{HF} / 6-311 \mathrm{G} * * \mathrm{E}(\mathrm{RHF})=-477.495537592$

C6H6-C5H5N $1 \mathrm{~b}(\mathrm{R}=5.0 \AA$ Å)

\begin{tabular}{|c|c|c|c|c|c|}
\hline \multirow{2}{*}{$\begin{array}{l}\text { Center } \\
\text { Number }\end{array}$} & \multirow{2}{*}{$\begin{array}{l}\text { Atomic } \\
\text { Number }\end{array}$} & \multicolumn{2}{|c|}{ Atomic } & \multicolumn{2}{|c|}{ Coordinates (Angstroms) } \\
\hline & & & Type & $X$ & $\mathrm{Z}$ \\
\hline 1 & 6 & 0 & -1.399120 & 0.000000 & -2.490920 \\
\hline 2 & 6 & 0 & -0.699560 & 1.211673 & -2.490920 \\
\hline 3 & 6 & 0 & 0.699560 & 1.211673 & -2.490920 \\
\hline 4 & 6 & 0 & 1.399120 & -0.000000 & -2.490920 \\
\hline 5 & 6 & 0 & 0.699560 & -1.211673 & -2.490920 \\
\hline 6 & 6 & 0 & -0.699560 & -1.211673 & -2.490920 \\
\hline 7 & 1 & 0 & -2.485527 & 0.000000 & -2.490920 \\
\hline 8 & 1 & 0 & -1.242763 & 2.152530 & -2.490920 \\
\hline 9 & 1 & 0 & 1.242763 & 2.152530 & -2.490920 \\
\hline 10 & 1 & 0 & 2.485527 & -0.000000 & -2.490920 \\
\hline 11 & 1 & 0 & 1.242763 & -2.152530 & -2.490920 \\
\hline 12 & 1 & 0 & -1.242763 & -2.152530 & -2.490920 \\
\hline 13 & 6 & 0 & -0.000000 & -0.000000 & 1.098980 \\
\hline 14 & 6 & 0 & 0.000000 & 1.197974 & 1.816470 \\
\hline 15 & 6 & 0 & 0.000000 & 1.143425 & 3.213605 \\
\hline
\end{tabular}




$\begin{array}{rrrrrr}16 & 6 & 0 & -0.000000 & -1.197974 & 1.816470 \\ 17 & 6 & 0 & -0.000000 & -1.143425 & 3.213605 \\ 18 & 7 & 0 & -0.000000 & -0.000000 & 3.919180 \\ 19 & 1 & 0 & -0.000000 & -0.000000 & 0.012880 \\ 20 & 1 & 0 & 0.000000 & 2.157849 & 1.309353 \\ 21 & 1 & 0 & 0.000000 & 2.060513 & 3.799005 \\ 22 & 1 & 0 & -0.000000 & -2.157849 & 1.309353 \\ 23 & 1 & 0 & -0.000000 & -2.060513 & 3.799005\end{array}$

$\mathrm{HF} / 6-311 \mathrm{G}^{* *} \mathrm{E}(\mathrm{RHF})=-477.500154193$

C6H6-C5H5N 1c (R = 4.6 Å)

\begin{tabular}{|c|c|c|c|c|c|}
\hline \multirow{2}{*}{$\begin{array}{l}\text { Center } \\
\text { Number }\end{array}$} & \multirow{2}{*}{$\begin{array}{l}\text { Atomic } \\
\text { Number }\end{array}$} & \multicolumn{2}{|c|}{ Atomic } & \multicolumn{2}{|c|}{ Coordinates (Angstroms } \\
\hline & & & Type & $X$ & $\mathrm{Z}$ \\
\hline 1 & 6 & 0 & -1.399120 & 0.000000 & -2.309080 \\
\hline 2 & 6 & 0 & -0.699560 & 1.211673 & -2.309080 \\
\hline 3 & 6 & 0 & 0.699560 & 1.211673 & -2.309080 \\
\hline 4 & 6 & 0 & 1.399120 & -0.000000 & -2.309080 \\
\hline 5 & 6 & 0 & 0.699560 & -1.211673 & -2.309080 \\
\hline 6 & 6 & 0 & -0.699560 & -1.211673 & -2.309080 \\
\hline 7 & 1 & 0 & -2.485527 & 0.000000 & -2.309080 \\
\hline 8 & 1 & 0 & -1.242763 & 2.152530 & -2.309080 \\
\hline 9 & 1 & 0 & 1.242763 & 2.152530 & -2.309080 \\
\hline 10 & 1 & 0 & 2.485527 & -0.000000 & -2.309080 \\
\hline 11 & 1 & 0 & 1.242763 & -2.152530 & -2.309080 \\
\hline 12 & 1 & 0 & -1.242763 & -2.152530 & -2.309080 \\
\hline 13 & 6 & 0 & 0.000000 & -0.000000 & 3.701020 \\
\hline 14 & 6 & 0 & -0.000000 & -1.197974 & 2.983530 \\
\hline 15 & 6 & 0 & -0.000000 & -1.143425 & 1.586395 \\
\hline 16 & 6 & 0 & 0.000000 & 1.197974 & 2.983530 \\
\hline 17 & 6 & 0 & 0.000000 & 1.143425 & 1.586395 \\
\hline 18 & 7 & 0 & 0.000000 & -0.000000 & 0.880820 \\
\hline 19 & 1 & 0 & 0.000000 & -0.000000 & 4.787120 \\
\hline 20 & 1 & 0 & -0.000000 & -2.157849 & 3.490647 \\
\hline 21 & 1 & 0 & -0.000000 & -2.060513 & 1.000995 \\
\hline 22 & 1 & 0 & 0.000000 & 2.157849 & 3.490647 \\
\hline 23 & 1 & 0 & 0.000000 & 2.060513 & 1.000995 \\
\hline
\end{tabular}

$\mathrm{HF} / 6-311 \mathrm{G} * * \mathrm{E}(\mathrm{RHF})=-477.494289978$

C6H6-C5H5N 1d (R = 5.0 ̊̊)

\begin{tabular}{lllllc} 
Center & \multicolumn{2}{c}{ Atomic } & \multicolumn{2}{c}{ Atomic } & \multicolumn{2}{c}{ Coordinates } & (Angstroms) \\
Number & Number & Type & X & Y & Z \\
-1 & 6 & 0 & 2.500000 & -0.009080 & 1.399120
\end{tabular}




$\begin{array}{cccccc}2 & 6 & 0 & 2.500000 & -1.220754 & 0.699560 \\ 3 & 6 & 0 & 2.500000 & -1.220754 & -0.699560 \\ 4 & 6 & 0 & 2.500000 & -0.009080 & -1.399120 \\ 5 & 6 & 0 & 2.500000 & 1.202593 & -0.699560 \\ 6 & 6 & 0 & 2.500000 & 1.202593 & 0.699560 \\ 7 & 1 & 0 & 2.500000 & -0.009080 & 2.485527 \\ 8 & 1 & 0 & 2.500000 & -2.161610 & 1.242763 \\ 9 & 1 & 0 & 2.500000 & -2.161610 & -1.242763 \\ 10 & 1 & 0 & 2.500000 & -0.009080 & -2.485527 \\ 11 & 1 & 0 & 2.500000 & 2.143449 & -1.242763 \\ 12 & 1 & 0 & 2.500000 & 2.143449 & 1.242763 \\ 13 & 6 & 0 & -2.500000 & 1.401020 & -0.000000 \\ 14 & 6 & 0 & -1.302026 & 0.683530 & 0.000000 \\ 15 & 6 & 0 & -1.356575 & -0.713605 & 0.000000 \\ 16 & 6 & 0 & -3.697974 & 0.683530 & 0.000000 \\ 17 & 6 & 0 & -3.643425 & -0.713605 & 0.000000 \\ 18 & 7 & 0 & -2.500000 & -1.419180 & -0.000000 \\ 19 & 1 & 0 & -2.500000 & 2.487120 & -0.000000 \\ 20 & 1 & 0 & -0.342151 & 1.190647 & -0.000000 \\ 21 & 1 & 0 & -0.439487 & -1.299005 & -0.000000 \\ 22 & 1 & 0 & -4.657849 & 1.190647 & -0.000000 \\ 23 & 1 & 0 & -4.560513 & -1.299005 & -0.000000 \\ ----------------------------------------------------------------\end{array}$

$\mathrm{HF} / 6-311 \mathrm{G} * * \mathrm{E}(\mathrm{RHF})=-477.499593118$

C6H6-C5H5N 1e (R = 5.0 ̊̊)

\begin{tabular}{|c|c|c|c|c|c|}
\hline \multirow{2}{*}{$\begin{array}{l}\text { Center } \\
\text { Number }\end{array}$} & \multirow{2}{*}{$\begin{array}{l}\text { Atomic } \\
\text { Number }\end{array}$} & \multicolumn{2}{|c|}{ Atomic } & \multicolumn{2}{|c|}{ Coordinates (Angstroms) } \\
\hline & & & Type & $\mathrm{Y}$ & $\mathrm{Z}$ \\
\hline 1 & 6 & 0 & 0.009080 & 1.100880 & -0.000000 \\
\hline 1 & 6 & & & 1.800440 & \\
\hline 3 & 6 & 0 & & 100560 & 211673 \\
\hline 4 & 6 & & & & \\
\hline 5 & 6 & & & 199560 & -1.211673 \\
\hline 6 & 6 & & & 40 & \\
\hline 7 & 1 & & 0.00 & 0.014473 & -0.0 \\
\hline 8 & 1 & & & & \\
\hline 9 & 1 & 0 & & & \\
\hline 10 & 1 & & & & \\
\hline 11 & 1 & 0 & & & \\
\hline 12 & 1 & & 0 & 1.2 & -2.152 \\
\hline 13 & 6 & 0 & & -2.5 & \\
\hline 14 & 6 & 0 & -0.6 & -2.500000 & -1.197974 \\
\hline 15 & 6 & 0 & & & -1.143425 \\
\hline 16 & 6 & 0 & -0.6 & -2.500 & 1.197974 \\
\hline 17 & 6 & 0 & & -2.500000 & 1.143425 \\
\hline 18 & 7 & 0 & & -2.500000 & -0.000000 \\
\hline 19 & 1 & 0 & -2.487120 & -2.500000 & 0.00000 \\
\hline 20 & 1 & 0 & -1.190647 & -2.500000 & -2.15784 \\
\hline 21 & 1 & 0 & 1.299005 & -2.500000 & -2.060513 \\
\hline
\end{tabular}




$\begin{array}{rrrrrr}22 & 1 & 0 & -1.190647 & -2.500000 & 2.157849 \\ 23 & 1 & 0 & 1.299005 & -2.500000 & 2.060513\end{array}$

$\mathrm{HF} / 6-311 \mathrm{G} * * \mathrm{E}(\mathrm{RHF})=-477.498326474$

C6H6-C5H5N $1 f$

\begin{tabular}{ccccccc} 
Center & \multicolumn{2}{c}{ Atomic } & Atomic & \multicolumn{3}{c}{ Coordinates } \\
Number & Number & Type & X & Y & Z \\
- & Nums & \\
1 & 6 & 0 & 1.593828 & 0.898450 & 0.000000 \\
2 & 6 & 0 & 1.068948 & 1.361880 & 1.210970 \\
3 & 6 & 0 & 0.020868 & 2.288770 & 1.211590 \\
4 & 6 & 0 & -0.506892 & 2.748750 & 0.000000 \\
5 & 6 & 0 & 0.020868 & 2.288770 & -1.211590 \\
6 & 6 & 0 & 1.068948 & 1.361880 & -1.210970 \\
7 & 1 & 0 & 2.401298 & 0.170980 & 0.000000 \\
8 & 1 & 0 & 1.479128 & 1.003710 & 2.151890 \\
9 & 1 & 0 & -0.383992 & 2.651680 & 2.153280 \\
10 & 1 & 0 & -1.318342 & 3.472580 & 0.000000 \\
11 & 1 & 0 & -0.383992 & 2.651680 & -2.153280 \\
12 & 1 & 0 & 1.479128 & 1.003710 & -2.151890 \\
13 & 6 & 0 & -1.635362 & -0.959980 & 0.000000 \\
14 & 6 & 0 & -1.073532 & -1.406470 & -1.196980 \\
15 & 6 & 0 & 0.020868 & -2.274390 & -1.143150 \\
16 & 6 & 0 & -1.073532 & -1.406470 & 1.196980 \\
17 & 6 & 0 & 0.020868 & -2.274390 & 1.143150 \\
18 & 7 & 0 & 0.575668 & -2.711950 & 0.000000 \\
19 & 1 & 0 & -2.479092 & -0.275460 & 0.000000 \\
20 & 1 & 0 & -1.469642 & -1.088340 & -2.157060 \\
21 & 1 & 0 & 0.480108 & -2.639670 & -2.060200 \\
22 & 1 & 0 & -1.469642 & -1.088340 & 2.157060 \\
23 & 1 & 0 & 0.480108 & -2.639670 & 2.060200 \\
-------------------------------------------------------
\end{tabular}

$\mathrm{HF} / 6-31 \mathrm{G}^{*} \quad \mathrm{E}(\mathrm{RHF})=-477.391871959$

C6H6-C5H5N $1 \mathrm{~g}$

\begin{tabular}{|c|c|c|c|c|}
\hline \multirow{2}{*}{$\begin{array}{l}\text { Center } \\
\text { Number }\end{array}$} & \multirow{2}{*}{$\begin{array}{l}\text { Atomic } \\
\text { Numbe }\end{array}$} & Atomic & \multicolumn{2}{|c|}{ Coordinates (Angstroms) } \\
\hline & & Type & $X$ & Z \\
\hline 1 & 6 & -0.687870 & 1.758930 & 0.000000 \\
\hline 2 & 6 & 0.011660 & 1.776120 & 1.211240 \\
\hline 3 & 6 & 1.410130 & 1.809030 & 1.211280 \\
\hline 4 & 6 & 2.109400 & 1.822100 & 0.000000 \\
\hline 5 & 6 & 1.410130 & 1.809030 & -1.211280 \\
\hline 6 & 6 & 0.011660 & 1.776120 & -1.211240 \\
\hline
\end{tabular}




$\begin{array}{cccrcc}7 & 1 & 0 & -1.774480 & 1.728980 & 0.000000 \\ 8 & 1 & 0 & -0.532280 & 1.764560 & 2.152650 \\ 9 & 1 & 0 & 1.954230 & 1.819100 & 2.152510 \\ 10 & 1 & 0 & 3.196240 & 1.840530 & 0.000000 \\ 11 & 1 & 0 & 1.954230 & 1.819100 & -2.152510 \\ 12 & 1 & 0 & -0.532280 & 1.764560 & -2.152650 \\ 13 & 6 & 0 & -2.102850 & -1.776820 & 0.000000 \\ 14 & 6 & 0 & -1.385170 & -1.781530 & 1.197550 \\ 15 & 6 & 0 & 0.011660 & -1.798390 & 1.142780 \\ 16 & 6 & 0 & -1.385170 & -1.781530 & -1.197550 \\ 17 & 6 & 0 & 0.011660 & -1.798390 & -1.142780 \\ 18 & 7 & 0 & 0.717220 & -1.813140 & 0.000000 \\ 19 & 1 & 0 & -3.189960 & -1.769140 & 0.000000 \\ 20 & 1 & 0 & -1.892530 & -1.776880 & 2.158290 \\ 21 & 1 & 0 & 0.598700 & -1.805000 & 2.059350 \\ 22 & 1 & 0 & -1.892530 & -1.776880 & -2.158290 \\ 23 & 1 & 0 & 0.598700 & -1.805000 & -2.059350 \\ ------------------------------\end{array}$

$\mathrm{HF} / 6-31 \mathrm{G}^{*} \quad \mathrm{E}(\mathrm{RHF})=-477.391435933$

C6H6-C5H5N $1 \mathrm{~h}$

\begin{tabular}{|c|c|c|c|c|c|}
\hline \multirow{2}{*}{$\begin{array}{l}\text { Center } \\
\text { Number }\end{array}$} & \multirow{2}{*}{\multicolumn{2}{|c|}{$\begin{array}{c}\text { Atomic } \\
\text { Number }\end{array}$}} & Atomic & \multicolumn{2}{|c|}{ Coordinates (Angstroms) } \\
\hline & & & Type & $\mathrm{X}$ & $\mathrm{Z}$ \\
\hline 1 & 6 & 0 & 2.459310 & 0.402760 & 1.399580 \\
\hline 2 & 6 & 0 & 2.459310 & -0.809110 & 0.699790 \\
\hline 3 & 6 & 0 & 2.459310 & -0.809110 & -0.699790 \\
\hline 4 & 6 & 0 & 2.459310 & 0.402760 & -1.399580 \\
\hline 5 & 6 & 0 & 2.461050 & 1.614910 & -0.699870 \\
\hline 6 & 6 & 0 & 2.461050 & 1.614910 & 0.699870 \\
\hline 7 & 1 & 0 & 2.458780 & 0.402470 & 2.486540 \\
\hline 8 & 1 & 0 & 2.461060 & -1.750210 & 1.243920 \\
\hline 9 & 1 & 0 & 2.461060 & -1.750210 & -1.243920 \\
\hline 10 & 1 & 0 & 2.458780 & 0.402470 & -2.486540 \\
\hline 11 & 1 & 0 & 2.465080 & 2.556180 & -1.243730 \\
\hline 12 & 1 & 0 & 2.465080 & 2.556180 & 1.243730 \\
\hline 13 & 6 & 0 & -2.317310 & 0.980380 & 0.000000 \\
\hline 14 & 6 & 0 & -1.197020 & 0.146350 & 0.000000 \\
\hline 15 & 6 & 0 & -1.397140 & -1.237720 & 0.000000 \\
\hline 16 & 6 & 0 & -3.583410 & 0.390470 & 0.000000 \\
\hline 17 & 6 & 0 & -3.672780 & -1.004620 & 0.000000 \\
\hline 18 & 7 & 0 & -2.607030 & -1.823870 & 0.000000 \\
\hline 19 & 1 & 0 & -2.206070 & 2.061780 & 0.000000 \\
\hline 20 & 1 & 0 & -0.189430 & 0.550120 & 0.000000 \\
\hline 21 & 1 & 0 & -0.543790 & -1.912630 & 0.000000 \\
\hline 22 & 1 & 0 & -4.486790 & 0.993880 & 0.000000 \\
\hline 23 & 1 & 0 & -4.644660 & -1.494830 & 0.000000 \\
\hline
\end{tabular}

$\mathrm{HF} / 6-31 \mathrm{G}^{*} \quad \mathrm{E}(\mathrm{RHF})=-477.395751541$ 
C6H6-C5H5N $1 \mathrm{i}$

\begin{tabular}{|c|c|c|c|c|c|}
\hline \multirow{2}{*}{$\begin{array}{l}\text { Center } \\
\text { Number }\end{array}$} & \multirow{2}{*}{$\begin{array}{l}\text { Atomic } \\
\text { Number }\end{array}$} & \multicolumn{2}{|c|}{ Atomic } & \multicolumn{2}{|c|}{ Coordinates (Angstroms) } \\
\hline & & & Type & $X$ & Z \\
\hline 1 & 6 & 0 & -1.399620 & 0.000000 & -2.529939 \\
\hline 2 & 6 & 0 & -0.699830 & 1.212010 & -2.530379 \\
\hline 3 & 6 & 0 & 0.699830 & 1.212010 & -2.530379 \\
\hline 4 & 6 & 0 & 1.399620 & 0.000000 & -2.529939 \\
\hline 5 & 6 & 0 & 0.699830 & -1.212010 & -2.530379 \\
\hline 6 & 6 & 0 & -0.699830 & -1.212010 & -2.530379 \\
\hline 7 & 1 & 0 & -2.486550 & 0.000000 & -2.529629 \\
\hline 8 & 1 & 0 & -1.243760 & 2.153190 & -2.533729 \\
\hline 9 & 1 & 0 & 1.243760 & 2.153190 & -2.533729 \\
\hline 10 & 1 & 0 & 2.486550 & 0.000000 & -2.529629 \\
\hline 11 & 1 & 0 & 1.243760 & -2.153190 & -2.533729 \\
\hline 12 & 1 & 0 & -1.243760 & -2.153190 & -2.533729 \\
\hline 13 & 6 & 0 & 0.000000 & 0.000000 & 1.136741 \\
\hline 14 & 6 & 0 & 0.000000 & 1.196850 & 1.856291 \\
\hline 15 & 6 & 0 & 0.000000 & 1.143520 & 3.253481 \\
\hline 16 & 6 & 0 & 0.000000 & -1.196850 & 1.856291 \\
\hline 17 & 6 & 0 & 0.000000 & -1.143520 & 3.253481 \\
\hline 18 & 7 & 0 & 0.000000 & 0.000000 & 3.959711 \\
\hline 19 & 1 & 0 & 0.000000 & 0.000000 & 0.051141 \\
\hline 20 & 1 & 0 & 0.000000 & 2.156540 & 1.347081 \\
\hline 21 & 1 & 0 & 0.000000 & 2.060040 & 3.840791 \\
\hline 22 & 1 & 0 & 0.000000 & -2.156540 & 1.347081 \\
\hline 23 & 1 & 0 & 0.000000 & -2.060040 & 3.840791 \\
\hline
\end{tabular}

$\mathrm{HF} / 6-31 \mathrm{G}^{*} \quad \mathrm{E}(\mathrm{RHF})=-477.396008638$

C6H6-C5H5N 1j

\begin{tabular}{cccccc} 
Center & \multicolumn{2}{c}{ Atomic } & \multicolumn{2}{c}{ Atomic } & \multicolumn{3}{c}{ Coordinates } & (Angstroms) \\
Number & Number & Type & X & Y & Z \\
-1 & 6 & 0 & -1.245550 & 0.063230 & 0.000000 \\
2 & 6 & 0 & -2.429500 & 0.808430 & 0.000000 \\
3 & 6 & 0 & -3.667280 & 0.155360 & 0.000000 \\
4 & 6 & 0 & -3.719830 & -1.242930 & 0.000000 \\
5 & 6 & 0 & -2.535080 & -1.987920 & 0.000000 \\
6 & 6 & 0 & -1.297280 & -1.335410 & 0.000000 \\
7 & 1 & 0 & -0.280440 & 0.561150 & 0.000000 \\
8 & 1 & 0 & -2.388620 & 1.895060 & 0.000000 \\
9 & 1 & 0 & -4.587870 & 0.733830 & 0.000000 \\
10 & 1 & 0 & -4.681380 & -1.750370 & 0.000000 \\
11 & 1 & 0 & -2.576720 & -3.074360 & 0.000000 \\
12 & 1 & 0 & -0.372820 & -1.907520 & 0.000000
\end{tabular}




$\begin{array}{rrrrrr}13 & 6 & 0 & 2.474870 & 1.982920 & 0.000000 \\ 14 & 6 & 0 & 2.485140 & 1.264130 & 1.197230 \\ 15 & 6 & 0 & 2.485140 & -0.133020 & 1.143980 \\ 16 & 6 & 0 & 2.485140 & 1.264130 & -1.197230 \\ 17 & 6 & 0 & 2.485140 & -0.133020 & -1.143980 \\ 18 & 7 & 0 & 2.485660 & -0.840030 & 0.000000 \\ 19 & 1 & 0 & 2.471970 & 3.069470 & 0.000000 \\ 20 & 1 & 0 & 2.464180 & 1.773680 & 2.156460 \\ 21 & 1 & 0 & 2.481200 & -0.714890 & 2.063580 \\ 22 & 1 & 0 & 2.464180 & 1.773680 & -2.156460 \\ 23 & 1 & 0 & 2.481200 & -0.714890 & -2.063580\end{array}$

$\mathrm{HF} / 6-31 \mathrm{G}^{*} \quad \mathrm{E}(\mathrm{RHF})=-477.395700786$

C6H6-C5H5NH 2a (R = $3.6 \AA)$

\begin{tabular}{|c|c|c|c|c|c|}
\hline \multirow{2}{*}{$\begin{array}{l}\text { Center } \\
\text { Number }\end{array}$} & \multirow{2}{*}{$\begin{array}{l}\text { Atomic } \\
\text { Number }\end{array}$} & \multicolumn{2}{|c|}{ Atomic } & \multicolumn{2}{|c|}{ Coordinates (Angstroms) } \\
\hline & & & Type & $X$ & $\mathrm{Z}$ \\
\hline 1 & 6 & 0 & 1.821176 & 1.427820 & 0.000000 \\
\hline 2 & 6 & 0 & 1.821176 & 0.728260 & 1.211673 \\
\hline 3 & 6 & 0 & 1.821176 & -0.670860 & 1.211673 \\
\hline 4 & 6 & 0 & 1.821176 & -1.370420 & 0.000000 \\
\hline 5 & 6 & 0 & 1.821176 & -0.670860 & -1.211673 \\
\hline 6 & 6 & 0 & 1.821176 & 0.728260 & -1.211673 \\
\hline 7 & 1 & 0 & 1.821176 & 2.514227 & 0.000000 \\
\hline 8 & 1 & 0 & 1.821176 & 1.271463 & 2.152530 \\
\hline 9 & 1 & 0 & 1.821176 & -1.214064 & 2.152530 \\
\hline 10 & 1 & 0 & 1.821176 & -2.456827 & 0.000000 \\
\hline 11 & 1 & 0 & 1.821176 & -1.214064 & -2.152530 \\
\hline 12 & 1 & 0 & 1.821176 & 1.271463 & -2.152530 \\
\hline 13 & 6 & 0 & -1.778824 & 1.392200 & 0.000000 \\
\hline 14 & 6 & 0 & -1.778824 & 0.690080 & 1.209822 \\
\hline 15 & 6 & 0 & -1.778824 & -0.699200 & 1.191633 \\
\hline 16 & 6 & 0 & -1.778824 & 0.690080 & -1.209822 \\
\hline 17 & 6 & 0 & -1.778824 & -0.699200 & -1.191633 \\
\hline 18 & 7 & 0 & -1.778824 & -1.334800 & 0.000000 \\
\hline 19 & 1 & 0 & -1.778824 & 2.477700 & 0.000000 \\
\hline 20 & 1 & 0 & -1.778824 & 1.205716 & 2.163216 \\
\hline 21 & 1 & 0 & -1.778824 & -1.320790 & 2.079223 \\
\hline 22 & 1 & 0 & -1.778824 & 1.205716 & -2.163216 \\
\hline 23 & 1 & 0 & -1.778824 & -1.320790 & -2.079223 \\
\hline 24 & 1 & 0 & -1.778824 & -2.353100 & 0.000000 \\
\hline
\end{tabular}

$\mathrm{HF} / 6-311 \mathrm{G}^{* *} \mathrm{E}(\mathrm{RHF})=-477.878663005$

C6H6-C5H5NH 2b (R = $4.6 \AA$ ) 


\begin{tabular}{|c|c|c|c|c|c|}
\hline \multirow{2}{*}{$\begin{array}{l}\text { Center } \\
\text { Number }\end{array}$} & Atomic & \multicolumn{2}{|c|}{ Atomic } & \multicolumn{2}{|c|}{ Coordinates (Angstroms) } \\
\hline & Numbe & & Type & $\mathrm{X}$ & $\mathrm{Z}$ \\
\hline 1 & 6 & 0 & -1.399120 & 0.000000 & -2.355759 \\
\hline 2 & 6 & 0 & -0.699560 & 1.211673 & -2.355759 \\
\hline 3 & 6 & 0 & 0.699560 & 1.211673 & -2.355759 \\
\hline 4 & 6 & 0 & 1.399120 & 0.000000 & -2.355759 \\
\hline 5 & 6 & 0 & 0.699560 & -1.211673 & -2.355759 \\
\hline 6 & 6 & 0 & -0.699560 & -1.211673 & -2.355759 \\
\hline 7 & 1 & 0 & -2.485527 & 0.000000 & -2.355759 \\
\hline 8 & 1 & 0 & -1.242763 & 2.152530 & -2.355759 \\
\hline 9 & 1 & 0 & 1.242763 & 2.152530 & -2.355759 \\
\hline 10 & 1 & 0 & 2.485527 & 0.000000 & -2.355759 \\
\hline 11 & 1 & 0 & 1.242763 & -2.152530 & -2.355759 \\
\hline 12 & 1 & 0 & -1.242763 & -2.152530 & -2.355759 \\
\hline 13 & 6 & 0 & 0.000000 & 0.000000 & 0.880741 \\
\hline 14 & 6 & 0 & 0.000000 & 1.209822 & 1.582861 \\
\hline 15 & 6 & 0 & 0.000000 & 1.191633 & 2.972142 \\
\hline 16 & 6 & 0 & 0.000000 & -1.209822 & 1.582861 \\
\hline 17 & 6 & 0 & 0.000000 & -1.191633 & 2.972142 \\
\hline 18 & 7 & 0 & 0.000000 & 0.000000 & 3.607741 \\
\hline 19 & 1 & 0 & 0.000000 & 0.000000 & -0.204759 \\
\hline 20 & 1 & 0 & 0.000000 & 2.163216 & 1.067225 \\
\hline 21 & 1 & 0 & 0.000000 & 2.079223 & 3.593731 \\
\hline 22 & 1 & 0 & 0.000000 & -2.163216 & 1.067225 \\
\hline 23 & 1 & 0 & 0.000000 & -2.079223 & 3.593731 \\
\hline 24 & 1 & 0 & 0.000000 & 0.000000 & 4.626041 \\
\hline
\end{tabular}

$\mathrm{HF} / 6-311 \mathrm{G} * * \mathrm{E}(\mathrm{RHF})=-477.882490229$

C6H6-C5H5NH 2c (R = $4.4 \AA)$

\begin{tabular}{|c|c|c|c|c|c|}
\hline \multirow{2}{*}{$\begin{array}{l}\text { Center } \\
\text { Number }\end{array}$} & Atomic & \multicolumn{2}{|c|}{ Atomic } & \multicolumn{2}{|c|}{ Coordinates (Angstroms) } \\
\hline & Number & & Type & $\mathrm{X}$ & $\mathrm{Z}$ \\
\hline 1 & 6 & 0 & -1.399120 & 0.000000 & -2.197183 \\
\hline 2 & 6 & 0 & -0.699560 & 1.211673 & 183 \\
\hline 3 & 6 & 0 & 0.699560 & 1.211673 & -2.197183 \\
\hline 4 & 6 & 0 & 1.399120 & 0.000000 & -2.197183 \\
\hline 5 & 6 & 0 & 0.699560 & -1.211673 & -2.197183 \\
\hline 6 & 6 & 0 & -0.699560 & -1.211673 & -2.197183 \\
\hline 7 & 1 & 0 & -2.485527 & 0.000000 & -2.197183 \\
\hline 8 & 1 & 0 & -1.242763 & 2.152530 & -2.197183 \\
\hline 9 & 1 & 0 & 1.242763 & 2.152530 & -2.197183 \\
\hline 10 & 1 & 0 & 2.485527 & 0.000000 & -2.197183 \\
\hline 11 & 1 & 0 & 1.242763 & -2.152530 & -2.197183 \\
\hline 12 & 1 & 0 & -1.242763 & -2.152530 & -2.197183 \\
\hline 13 & 6 & 0 & 0.000000 & 0.000000 & 3.566317 \\
\hline 14 & 6 & 0 & 0.000000 & -1.209822 & 2.864198 \\
\hline 15 & 6 & 0 & 0.000000 & -1.191633 & 1.474917 \\
\hline 16 & 6 & 0 & 0.000000 & 1.209822 & 2.864198 \\
\hline
\end{tabular}




$\begin{array}{llllll}17 & 6 & 0 & 0.000000 & 1.191633 & 1.474917 \\ 18 & 7 & 0 & 0.000000 & 0.000000 & 0.839317 \\ 19 & 1 & 0 & 0.000000 & 0.000000 & 4.651817 \\ 20 & 1 & 0 & 0.000000 & -2.163216 & 3.379834 \\ 21 & 1 & 0 & 0.000000 & -2.079223 & 0.853328 \\ 22 & 1 & 0 & 0.000000 & 2.163216 & 3.379834 \\ 23 & 1 & 0 & 0.000000 & 2.079223 & 0.853328 \\ 24 & 1 & 0 & 0.000000 & 0.000000 & -0.178983\end{array}$

$\mathrm{HF} / 6-311 \mathrm{G}^{* *} \mathrm{E}(\mathrm{RHF})=-477.890159646$

\begin{tabular}{|c|c|c|c|c|c|}
\hline \multirow{2}{*}{$\begin{array}{l}\text { Center } \\
\text { Number }\end{array}$} & \multirow{2}{*}{\multicolumn{2}{|c|}{$\begin{array}{l}\text { Atomic } \\
\text { Number }\end{array}$}} & Atomic & \multicolumn{2}{|c|}{ Coordinates (Angstroms) } \\
\hline & & & Type & $X$ & Z \\
\hline 1 & 6 & 0 & 2.428235 & 0.028700 & 1.399120 \\
\hline 2 & 6 & 0 & 2.428235 & -1.182974 & 0.699560 \\
\hline 3 & 6 & 0 & 2.428235 & -1.182974 & -0.699560 \\
\hline 4 & 6 & 0 & 2.428235 & 0.028700 & -1.399120 \\
\hline 5 & 6 & 0 & 2.428235 & 1.240373 & -0.699560 \\
\hline 6 & 6 & 0 & 2.428235 & 1.240373 & 0.699560 \\
\hline 7 & 1 & 0 & 2.428235 & 0.028700 & 2.485527 \\
\hline 8 & 1 & 0 & 2.428235 & -2.123830 & 1.242764 \\
\hline 9 & 1 & 0 & 2.428235 & -2.123830 & -1.242764 \\
\hline 10 & 1 & 0 & 2.428235 & 0.028700 & -2.485527 \\
\hline 11 & 1 & 0 & 2.428235 & 2.181229 & -1.242764 \\
\hline 12 & 1 & 0 & 2.428235 & 2.181229 & 1.242764 \\
\hline 13 & 6 & 0 & -2.371765 & 1.392200 & 0.000000 \\
\hline 14 & 6 & 0 & -1.161942 & 0.690080 & 0.000000 \\
\hline 15 & 6 & 0 & -1.180132 & -0.699200 & 0.000000 \\
\hline 16 & 6 & 0 & -3.581587 & 0.690080 & 0.000000 \\
\hline 17 & 6 & 0 & -3.563398 & -0.699200 & 0.000000 \\
\hline 18 & 7 & 0 & -2.371765 & -1.334800 & 0.000000 \\
\hline 19 & 1 & 0 & -2.371765 & 2.477700 & 0.000000 \\
\hline 20 & 1 & 0 & -0.208549 & 1.205716 & 0.000000 \\
\hline 21 & 1 & 0 & -0.292542 & -1.320790 & 0.000000 \\
\hline 22 & 1 & 0 & -4.534980 & 1.205716 & 0.000000 \\
\hline 23 & 1 & 0 & -4.450988 & -1.320790 & 0.000000 \\
\hline 24 & 1 & 0 & -2.371765 & -2.353100 & 0.000000 \\
\hline
\end{tabular}

$\mathrm{HF} / 6-311 \mathrm{G}^{* *} \mathrm{E}(\mathrm{RHF})=-477.883900935$

C6H6-C5H5NH 2e (R = 5.0 ̊̊)

\begin{tabular}{llll} 
Center Atomic Atomic & \multicolumn{2}{c}{ Coordinates (Angstroms) } \\
Number & Number Type & X & Y \\
-
\end{tabular}




$\begin{array}{cccccc}1 & 6 & 0 & 1.130292 & 0.028700 & 0.000000 \\ 2 & 6 & 0 & 1.829852 & 0.028700 & 1.211673 \\ 3 & 6 & 0 & 3.228972 & 0.028700 & 1.211673 \\ 4 & 6 & 0 & 3.928532 & 0.028700 & 0.000000 \\ 5 & 6 & 0 & 3.228972 & 0.028700 & -1.211673 \\ 6 & 6 & 0 & 1.829852 & 0.028700 & -1.211673 \\ 7 & 1 & 0 & 0.043885 & 0.028700 & 0.000000 \\ 8 & 1 & 0 & 1.286648 & 0.028700 & 2.152530 \\ 9 & 1 & 0 & 3.772175 & 0.028700 & 2.152530 \\ 10 & 1 & 0 & 5.014939 & 0.028700 & 0.000000 \\ 11 & 1 & 0 & 3.772175 & 0.028700 & -2.152530 \\ 12 & 1 & 0 & 1.286648 & 0.028700 & -2.152530 \\ 13 & 6 & 0 & -2.470588 & 1.392200 & 0.000000 \\ 14 & 6 & 0 & -2.470588 & 0.690080 & -1.209822 \\ 15 & 6 & 0 & -2.470588 & -0.699200 & -1.191633 \\ 16 & 6 & 0 & -2.470588 & 0.690080 & 1.209822 \\ 17 & 6 & 0 & -2.470588 & -0.699200 & 1.191633 \\ 18 & 7 & 0 & -2.470588 & -1.334800 & 0.000000 \\ 19 & 1 & 0 & -2.470588 & 2.477700 & 0.000000 \\ 20 & 1 & 0 & -2.470588 & 1.205716 & -2.163216 \\ 21 & 1 & 0 & -2.470588 & -1.320790 & -2.079223 \\ 22 & 1 & 0 & -2.470588 & 1.205716 & 2.163216 \\ 23 & 1 & 0 & -2.470588 & -1.320790 & 2.079223 \\ 24 & 1 & 0 & -2.470588 & -2.353100 & 0.000000 \\ ---------------------------------------------------------\end{array}$

$\mathrm{HF} / 6-311 \mathrm{G} * * \mathrm{E}(\mathrm{RHF})=-477.874461237$

\section{C6H6-C5H5NH $2 \mathrm{f}$}

\begin{tabular}{cccccc} 
Center & \multicolumn{2}{c}{ Atomic } & \multicolumn{2}{c}{ Atomic } & \multicolumn{3}{c}{ Coordinates } \\
Number & Number & Type & X & Y & Z \\
$----------1 r o m s)$ \\
1 & 6 & 0 & 1.567199 & -1.199560 & 0.000000 \\
2 & 6 & 0 & 0.934108 & -1.499430 & 1.213380 \\
3 & 6 & 0 & -0.333242 & -2.095289 & 1.212940 \\
4 & 6 & 0 & -0.967802 & -2.390458 & 0.000000 \\
5 & 6 & 0 & -0.333242 & -2.095289 & -1.212940 \\
6 & 6 & 0 & 0.934108 & -1.499430 & -1.213380 \\
7 & 1 & 0 & 2.573899 & -0.787271 & 0.000000 \\
8 & 1 & 0 & 1.445779 & -1.313070 & 2.154790 \\
9 & 1 & 0 & -0.808592 & -2.363038 & 2.153420 \\
10 & 1 & 0 & -1.935343 & -2.886317 & 0.000000 \\
11 & 1 & 0 & -0.808592 & -2.363038 & -2.153420 \\
12 & 1 & 0 & 1.445779 & -1.313070 & -2.154790 \\
13 & 6 & 0 & -1.487689 & 0.986433 & 0.000000 \\
14 & 6 & 0 & -0.894549 & 1.364342 & -1.207850 \\
15 & 6 & 0 & 0.266482 & 2.126571 & -1.189860 \\
16 & 6 & 0 & -0.894549 & 1.364342 & 1.207850 \\
17 & 6 & 0 & 0.266482 & 2.126571 & 1.189860 \\
18 & 7 & 0 & 0.796812 & 2.480590 & 0.000000 \\
19 & 1 & 0 & -2.397390 & 0.395553 & 0.000000
\end{tabular}




$\begin{array}{rrrrrr}20 & 1 & 0 & -1.325299 & 1.080322 & -2.161070 \\ 21 & 1 & 0 & 0.784602 & 2.466100 & -2.079090 \\ 22 & 1 & 0 & -1.325299 & 1.080322 & 2.161070 \\ 23 & 1 & 0 & 0.784602 & 2.466100 & 2.079090 \\ 24 & 1 & 0 & 1.644333 & 3.040459 & 0.000000\end{array}$

HF/6-31G* $\mathrm{E}(\mathrm{RHF})=-477.773082544$

\section{C6H6-C5H5NH 2g}

\begin{tabular}{|c|c|c|c|c|c|}
\hline \multirow{2}{*}{$\begin{array}{l}\text { Center } \\
\text { Number }\end{array}$} & \multirow{2}{*}{$\begin{array}{l}\text { Atomic } \\
\text { Number }\end{array}$} & \multicolumn{2}{|c|}{ Atomic } & \multicolumn{2}{|c|}{ Coordinates (Angstroms) } \\
\hline & & & Type & X & $\mathrm{Z}$ \\
\hline 1 & 6 & 0 & -1.171089 & 2.267941 & 0.000000 \\
\hline 2 & 6 & 0 & -0.472389 & 2.227441 & 1.213750 \\
\hline 3 & 6 & 0 & 0.927101 & 2.138521 & 1.215480 \\
\hline 4 & 6 & 0 & 1.628511 & 2.094241 & 0.000000 \\
\hline 5 & 6 & 0 & 0.927101 & 2.138521 & -1.215480 \\
\hline 6 & 6 & 0 & -0.472389 & 2.227441 & -1.213750 \\
\hline 7 & 1 & 0 & -2.253589 & 2.361521 & 0.000000 \\
\hline 8 & 1 & 0 & -1.013619 & 2.296111 & 2.153800 \\
\hline 9 & 1 & 0 & 1.473441 & 2.147841 & 2.155350 \\
\hline 10 & 1 & 0 & 2.714971 & 2.060231 & 0.000000 \\
\hline 11 & 1 & 0 & 1.473441 & 2.147841 & -2.155350 \\
\hline 12 & 1 & 0 & -1.013619 & 2.296111 & -2.153800 \\
\hline 13 & 6 & 0 & -0.716489 & -3.470309 & 0.000000 \\
\hline 14 & 6 & 0 & -0.472389 & -2.810809 & 1.208810 \\
\hline 15 & 6 & 0 & 0.010541 & -1.507529 & 1.186970 \\
\hline 16 & 6 & 0 & -0.472389 & -2.810809 & -1.208810 \\
\hline 17 & 6 & 0 & 0.010541 & -1.507529 & -1.186970 \\
\hline 18 & 7 & 0 & 0.230731 & -0.909259 & 0.000000 \\
\hline 19 & 1 & 0 & -1.093829 & -4.488469 & 0.000000 \\
\hline 20 & 1 & 0 & -0.651359 & -3.295289 & 2.162250 \\
\hline 21 & 1 & 0 & 0.224361 & -0.919749 & 2.071910 \\
\hline 22 & 1 & 0 & -0.651359 & -3.295289 & -2.162250 \\
\hline 23 & 1 & 0 & 0.224361 & -0.919749 & -2.071910 \\
\hline 24 & 1 & 0 & 0.591691 & 0.050981 & 0.000000 \\
\hline
\end{tabular}

$\mathrm{HF} / 6-31 \mathrm{G}^{*} \mathrm{E}(\mathrm{RHF})=-477.786353262$

\section{C6H6-C5H5NH 2h}

\begin{tabular}{llllll} 
Center & Atomic & Atomic & \multicolumn{3}{c}{ Coordinates (Angstroms) } \\
Number & Number & Type & X & Y & Z \\
-1 & 6 & 0 & -1.401040 & 0.000000 & -2.429499 \\
2 & 6 & 0 & -0.700530 & 1.213190 & -2.430789 \\
3 & 6 & 0 & 0.700530 & 1.213190 & -2.430789
\end{tabular}




$\begin{array}{cccccc}4 & 6 & 0 & 1.401040 & 0.000000 & -2.429499 \\ 5 & 6 & 0 & 0.700530 & -1.213190 & -2.430789 \\ 6 & 6 & 0 & -0.700530 & -1.213190 & -2.430789 \\ 7 & 1 & 0 & -2.487600 & 0.000000 & -2.454789 \\ 8 & 1 & 0 & -1.244700 & 2.153730 & -2.462899 \\ 9 & 1 & 0 & 1.244700 & 2.153730 & -2.462899 \\ 10 & 1 & 0 & 2.487600 & 0.000000 & -2.454789 \\ 11 & 1 & 0 & 1.244700 & -2.153730 & -2.462899 \\ 12 & 1 & 0 & -1.244700 & -2.153730 & -2.462899 \\ 13 & 6 & 0 & 0.000000 & 0.000000 & 0.955771 \\ 14 & 6 & 0 & 0.000000 & 1.208290 & 1.660341 \\ 15 & 6 & 0 & 0.000000 & 1.191010 & 3.049241 \\ 16 & 6 & 0 & 0.000000 & -1.208290 & 1.660341 \\ 17 & 6 & 0 & 0.000000 & -1.191010 & 3.049241 \\ 18 & 7 & 0 & 0.000000 & 0.000000 & 3.687071 \\ 19 & 1 & 0 & 0.000000 & 0.000000 & -0.129049 \\ 20 & 1 & 0 & 0.000000 & 2.159720 & 1.140121 \\ 21 & 1 & 0 & 0.000000 & 2.079340 & 3.670301 \\ 22 & 1 & 0 & 0.000000 & -2.159720 & 1.140121 \\ 23 & 1 & 0 & 0.000000 & -2.079340 & 3.670301 \\ 24 & 1 & 0 & 0.000000 & 0.000000 & 4.703201 \\ --------------------------------------------------------------------\end{array}$

$\mathrm{HF} / 6-31 \mathrm{G}^{*} \mathrm{E}(\mathrm{RHF})=-477.778552733$

C6H6-C5H5NH 2i

\begin{tabular}{cccccc} 
Center & \multicolumn{2}{c}{ Atomic } & \multicolumn{2}{c}{ Atomic } & \multicolumn{3}{c}{ Coordinates } & $($ Angstroms $)$ \\
Number & Number & Type & X & Y & $Z$ \\
-1 & 6 & 0 & -1.266818 & -0.068481 & 0.000000 \\
2 & 6 & 0 & -2.523608 & 0.546359 & 0.000000 \\
3 & 6 & 0 & -3.682578 & -0.236751 & 0.000000 \\
4 & 6 & 0 & -3.585558 & -1.632491 & 0.000000 \\
5 & 6 & 0 & -2.329658 & -2.248281 & 0.000000 \\
6 & 6 & 0 & -1.170948 & -1.464491 & 0.000000 \\
7 & 1 & 0 & -0.373118 & 0.552609 & 0.000000 \\
8 & 1 & 0 & -2.601638 & 1.630339 & 0.000000 \\
9 & 1 & 0 & -4.658848 & 0.239769 & 0.000000 \\
10 & 1 & 0 & -4.486418 & -2.239581 & 0.000000 \\
11 & 1 & 0 & -2.255478 & -3.332281 & 0.000000 \\
12 & 1 & 0 & -0.201078 & -1.959601 & 0.000000 \\
13 & 6 & 0 & 2.372432 & 2.251349 & 0.000000 \\
14 & 6 & 0 & 2.369212 & 1.549059 & 1.209590 \\
15 & 6 & 0 & 2.369212 & 0.159759 & 1.190790 \\
16 & 6 & 0 & 2.369212 & 1.549059 & -1.209590 \\
17 & 6 & 0 & 2.369212 & 0.159759 & -1.190790 \\
18 & 7 & 0 & 2.375572 & -0.476251 & 0.000000 \\
19 & 1 & 0 & 2.374212 & 3.337259 & 0.000000 \\
20 & 1 & 0 & 2.365072 & 2.064689 & 2.163540 \\
21 & 1 & 0 & 2.363342 & -0.460361 & 2.079780 \\
22 & 1 & 0 & 2.365072 & 2.064689 & -2.163540
\end{tabular}




$\begin{array}{cccccc}23 & 1 & 0 & 2.363342 & -0.460361 & -2.079780 \\ 24 & 1 & 0 & 2.375872 & -1.492501 & 0.000000\end{array}$

$\mathrm{HF} / 6-31 \mathrm{G} * \mathrm{E}(\mathrm{RHF})=-477.769831239$

C6H6-C5H5NCH3 3a (R = $3.6 \AA ̊$ )

\begin{tabular}{|c|c|c|c|c|c|}
\hline \multirow{2}{*}{$\begin{array}{l}\text { Center } \\
\text { Number }\end{array}$} & \multirow{2}{*}{\multicolumn{2}{|c|}{$\begin{array}{l}\text { Atomic } \\
\text { Number }\end{array}$}} & & \multicolumn{2}{|c|}{ Coordinates (Angstroms) } \\
\hline & & & Type & $X$ & $\mathrm{Z}$ \\
\hline 1 & 6 & 0 & -1.974059 & -1.685360 & 0.000000 \\
\hline 2 & 6 & 0 & -1.974059 & -0.985800 & 1.211673 \\
\hline 3 & 6 & 0 & -1.974059 & 0.413320 & 1.211673 \\
\hline 4 & 6 & 0 & -1.974059 & 1.112880 & 0.000000 \\
\hline 5 & 6 & 0 & -1.974059 & 0.413320 & -1.211673 \\
\hline 6 & 6 & 0 & -1.974059 & -0.985800 & -1.211673 \\
\hline 7 & 1 & 0 & -1.974059 & -2.771767 & 0.000000 \\
\hline 8 & 1 & 0 & -1.974059 & -1.529004 & 2.152530 \\
\hline 9 & 1 & 0 & -1.974059 & 0.956523 & 2.152530 \\
\hline 10 & 1 & 0 & -1.974059 & 2.199287 & 0.000000 \\
\hline 11 & 1 & 0 & -1.974059 & 0.956523 & -2.152530 \\
\hline 12 & 1 & 0 & -1.974059 & -1.529004 & -2.152530 \\
\hline 13 & 6 & 0 & 1.625941 & -1.666840 & 0.000000 \\
\hline 14 & 6 & 0 & 1.625941 & -0.956983 & 1.203325 \\
\hline 15 & 6 & 0 & 1.625941 & 0.432527 & 1.180376 \\
\hline 16 & 6 & 0 & 1.625941 & -0.956983 & -1.203325 \\
\hline 17 & 6 & 0 & 1.625941 & 0.432527 & -1.180376 \\
\hline 18 & 7 & 0 & 1.625941 & 1.094360 & 0.000000 \\
\hline 19 & 1 & 0 & 1.625941 & -2.751940 & 0.000000 \\
\hline 20 & 1 & 0 & 1.625941 & -1.464493 & 2.161408 \\
\hline 21 & 1 & 0 & 1.625941 & 1.041957 & 2.076721 \\
\hline 22 & 1 & 0 & 1.625941 & -1.464493 & -2.161408 \\
\hline 23 & 1 & 0 & 1.625941 & 1.041957 & -2.076721 \\
\hline 24 & 6 & 0 & 1.625941 & 2.578560 & 0.000000 \\
\hline 25 & 1 & 0 & 2.657855 & 2.932420 & 0.000000 \\
\hline 26 & 1 & 0 & 1.103715 & 2.924660 & -0.890249 \\
\hline 27 & 1 & 0 & 1.103715 & 2.924660 & 0.890249 \\
\hline
\end{tabular}

$\mathrm{HF} / 6-311 \mathrm{G} * * \mathrm{E}(\mathrm{RHF})=-516.921692267$

C6H6-C5H5NCH3 3b (R = $4.6 \AA ̊$ )

\begin{tabular}{lllrrr} 
Center & \multicolumn{2}{c}{ Atomic } & \multicolumn{2}{c}{ Atomic } & \multicolumn{2}{c}{ Coordinates } \\
Number & Number & Type & X & Y & Z \\
-1 & 6 & 0 & 1.399255 & -2.808821 & 0.000000 \\
2 & 6 & 0 & 0.699695 & -2.808821 & 1.211673 \\
3 & 6 & 0 & -0.699425 & -2.808821 & 1.211673
\end{tabular}




$\begin{array}{cccrcc}4 & 6 & 0 & -1.398985 & -2.808821 & 0.000000 \\ 5 & 6 & 0 & -0.699425 & -2.808821 & -1.211673 \\ 6 & 6 & 0 & 0.699695 & -2.808821 & -1.211673 \\ 7 & 1 & 0 & 2.485662 & -2.808821 & 0.000000 \\ 8 & 1 & 0 & 1.242898 & -2.808821 & 2.152530 \\ 9 & 1 & 0 & -1.242629 & -2.808821 & 2.152530 \\ 10 & 1 & 0 & -2.485392 & -2.808821 & 0.000000 \\ 11 & 1 & 0 & -1.242629 & -2.808821 & -2.152530 \\ 12 & 1 & 0 & 1.242898 & -2.808821 & -2.152530 \\ 13 & 6 & 0 & 0.000135 & 0.410579 & 0.000000 \\ 14 & 6 & 0 & 0.000135 & 1.120436 & 1.203325 \\ 15 & 6 & 0 & 0.000135 & 2.509947 & 1.180376 \\ 16 & 6 & 0 & 0.000135 & 1.120436 & -1.203325 \\ 17 & 6 & 0 & 0.000135 & 2.509947 & -1.180376 \\ 18 & 7 & 0 & 0.000135 & 3.171779 & 0.000000 \\ 19 & 1 & 0 & 0.000135 & -0.674521 & 0.000000 \\ 20 & 1 & 0 & 0.000135 & 0.612926 & 2.161408 \\ 21 & 1 & 0 & 0.000135 & 3.119376 & 2.076721 \\ 22 & 1 & 0 & 0.000135 & 0.612926 & -2.161408 \\ 23 & 1 & 0 & 0.000135 & 3.119376 & -2.076721 \\ 24 & 6 & 0 & 0.000135 & 4.655979 & 0.000000 \\ 25 & 1 & 0 & 1.032048 & 5.009840 & 0.000000 \\ 26 & 1 & 0 & -0.522092 & 5.002080 & -0.890249 \\ 27 & 1 & 0 & -0.522092 & 5.002080 & 0.890249 \\ ---------------------------------------------------------\end{array}$

$\mathrm{HF} / 6-311 \mathrm{G}^{* *} \mathrm{E}(\mathrm{RHF})=-516.925117246$

C6H6-C5H5NCH3 3c (R = $6.2 \AA)$

\begin{tabular}{cccccc} 
Center & \multicolumn{2}{c}{ Atomic } & Atomic & \multicolumn{3}{c}{ Coordinates } & $($ Angstroms) \\
Number & Number & Type & X & Y & Z \\
--1 & 6 & 0 & 1.399255 & -3.113760 & 0.000000 \\
2 & 6 & 0 & 0.699695 & -3.113760 & 1.211673 \\
3 & 6 & 0 & -0.699425 & -3.113760 & 1.211673 \\
4 & 6 & 0 & -1.398985 & -3.113760 & 0.000000 \\
5 & 6 & 0 & -0.699425 & -3.113760 & -1.211673 \\
6 & 6 & 0 & 0.699695 & -3.113760 & -1.211673 \\
7 & 1 & 0 & 2.485662 & -3.113760 & 0.000000 \\
8 & 1 & 0 & 1.242898 & -3.113760 & 2.152530 \\
9 & 1 & 0 & -1.242629 & -3.113760 & 2.152530 \\
10 & 1 & 0 & -2.485392 & -3.113760 & 0.000000 \\
11 & 1 & 0 & -1.242629 & -3.113760 & -2.152530 \\
12 & 1 & 0 & 1.242898 & -3.113760 & -2.152530 \\
13 & 6 & 0 & 0.000135 & 4.466840 & 0.000000 \\
14 & 6 & 0 & 0.000135 & 3.756983 & -1.203325 \\
15 & 6 & 0 & 0.000135 & 2.367473 & -1.180376 \\
16 & 6 & 0 & 0.000135 & 3.756983 & 1.203325 \\
17 & 6 & 0 & 0.000135 & 2.367473 & 1.180376 \\
18 & 7 & 0 & 0.000135 & 1.705640 & 0.000000 \\
19 & 1 & 0 & 0.000135 & 5.551940 & 0.000000
\end{tabular}




$\begin{array}{rrrrrr}20 & 1 & 0 & 0.000135 & 4.264493 & -2.161408 \\ 21 & 1 & 0 & 0.000135 & 1.758043 & -2.076721 \\ 22 & 1 & 0 & 0.000135 & 4.264493 & 2.161408 \\ 23 & 1 & 0 & 0.000135 & 1.758043 & 2.076721 \\ 24 & 6 & 0 & 0.000135 & 0.221440 & 0.000000 \\ 25 & 1 & 0 & 1.032048 & -0.132420 & 0.000000 \\ 26 & 1 & 0 & -0.522092 & -0.124660 & 0.890249 \\ 27 & 1 & 0 & -0.522092 & -0.124660 & -0.890249\end{array}$

$\mathrm{HF} / 6-311 \mathrm{G}^{* *} \mathrm{E}(\mathrm{RHF})=-516.925783165$

\begin{tabular}{|c|c|c|c|c|c|}
\hline \multirow{2}{*}{$\begin{array}{l}\text { Center } \\
\text { Number }\end{array}$} & \multirow{2}{*}{\multicolumn{2}{|c|}{$\begin{array}{l}\text { Atomic } \\
\text { Number }\end{array}$}} & Atomic & \multicolumn{2}{|c|}{ Coordinates (Angstroms) } \\
\hline & & & Type & X & Z \\
\hline 1 & 6 & 0 & -2.646732 & 0.076837 & 1.399120 \\
\hline 2 & 6 & 0 & -2.480662 & 1.277235 & 0.699834 \\
\hline 3 & 6 & 0 & -2.480582 & 1.277544 & -0.699286 \\
\hline 4 & 6 & 0 & -2.646571 & 0.077454 & -1.399120 \\
\hline 5 & 6 & 0 & -2.812641 & -1.122945 & -0.699835 \\
\hline 6 & 6 & 0 & -2.812722 & 3253 & 0.699285 \\
\hline 7 & 1 & 0 & -2.646794 & 0.076597 & 2.485527 \\
\hline 8 & 1 & 0 & -2.351772 & 2.209097 & 1.243251 \\
\hline 9 & 1 & 0 & -2.351629 & 2.209646 & -1.242276 \\
\hline 10 & 1 & 0 & -2.646509 & 0.077694 & -2.485527 \\
\hline 11 & 1 & 0 & -2.941531 & -2.054806 & -1.243251 \\
\hline 12 & 1 & 0 & -2.941674 & -2.055355 & 1.242276 \\
\hline 13 & 6 & 0 & 1.918896 & -1.948153 & -0.000185 \\
\hline 14 & 6 & 0 & 0.824189 & -1.080106 & -0.000056 \\
\hline 15 & 6 & 0 & 1.037319 & 0.293153 & 0.000259 \\
\hline 16 & 6 & 0 & 3.208139 & -1.409878 & 0.000008 \\
\hline 17 & 6 & 0 & 3.375804 & -0.030329 & 0.000322 \\
\hline 18 & 7 & 0 & 2.297250 & 0.787002 & 0.000441 \\
\hline 19 & 1 & 0 & 1.770210 & -3.023018 & -0.000431 \\
\hline 20 & 1 & 0 & -0.194399 & -1.451548 & -0.000197 \\
\hline 21 & 1 & 0 & 0.232936 & 1.019657 & 0.000373 \\
\hline 22 & 1 & 0 & 4.087643 & -2.043882 & -0.000081 \\
\hline 23 & 1 & 0 & 4.347202 & 0.450530 & 0.000484 \\
\hline 24 & 6 & 0 & 2.500622 & 2.257202 & 0.000777 \\
\hline 25 & 1 & 0 & 2.549051 & 2.607497 & 1.032770 \\
\hline 26 & 1 & 0 & 3.429929 & 2.478167 & -0.521348 \\
\hline 27 & 1 & 0 & 1.666225 & 2.722140 & -0.521395 \\
\hline
\end{tabular}

$\mathrm{HF} / 6-311 \mathrm{G}^{* *} \mathrm{E}(\mathrm{RHF})=-516.926939442$

C6H6-C5H5NCH3 3e (R = $5.0 \AA ̊)$ 


\begin{tabular}{|c|c|c|c|c|c|}
\hline \multirow{2}{*}{$\begin{array}{l}\text { Center } \\
\text { Number }\end{array}$} & Atomic & \multicolumn{2}{|c|}{ Atomic } & \multicolumn{2}{|c|}{ Coordinates (Angstroms) } \\
\hline & Numbe & & Type & $\mathrm{Y}$ & Z \\
\hline 1 & 6 & 0 & -1.342681 & -0.286240 & 0.000000 \\
\hline 2 & 6 & 0 & -2.042241 & -0.286240 & 1.211673 \\
\hline 3 & 6 & 0 & -3.441361 & -0.286240 & 1.211673 \\
\hline 4 & 6 & 0 & -4.140921 & -0.286240 & 0.000000 \\
\hline 5 & 6 & 0 & -3.441361 & -0.286240 & -1.211673 \\
\hline 6 & 6 & 0 & -2.042241 & -0.286240 & -1.211673 \\
\hline 7 & 1 & 0 & -0.256274 & -0.286240 & 0.000000 \\
\hline 8 & 1 & 0 & -1.499037 & -0.286240 & 2.152530 \\
\hline 9 & 1 & 0 & -3.984564 & -0.286240 & 2.152530 \\
\hline 10 & 1 & 0 & -5.227328 & -0.286240 & 0.000000 \\
\hline 11 & 1 & 0 & -3.984564 & -0.286240 & -2.152530 \\
\hline 12 & 1 & 0 & -1.499037 & -0.286240 & -2.152530 \\
\hline 13 & 6 & 0 & 2.258199 & -1.666840 & 0.000000 \\
\hline 14 & 6 & 0 & 2.258199 & -0.956983 & -1.203325 \\
\hline 15 & 6 & 0 & 2.258199 & 0.432527 & -1.180376 \\
\hline 16 & 6 & 0 & 2.258199 & -0.956983 & 1.203325 \\
\hline 17 & 6 & 0 & 2.258199 & 0.432527 & 1.180376 \\
\hline 18 & 7 & 0 & 2.258199 & 1.094360 & 0.000000 \\
\hline 19 & 1 & 0 & 2.258199 & -2.751940 & 0.000000 \\
\hline 20 & 1 & 0 & 2.258199 & -1.464493 & -2.161408 \\
\hline 21 & 1 & 0 & 2.258199 & 1.041957 & -2.076721 \\
\hline 22 & 1 & 0 & 2.258199 & -1.464493 & 2.161408 \\
\hline 23 & 1 & 0 & 2.258199 & 1.041957 & 2.076721 \\
\hline 24 & 6 & 0 & 2.258199 & 2.578560 & 0.000000 \\
\hline 25 & 1 & 0 & 3.290113 & 2.932420 & 0.000000 \\
\hline 26 & 1 & 0 & 1.735973 & 2.924660 & -0.890249 \\
\hline 27 & 1 & 0 & 1.735973 & 2.924660 & 0.890249 \\
\hline
\end{tabular}

$\mathrm{HF} / 6-311 \mathrm{G} * * \mathrm{E}(\mathrm{RHF})=-516.918416725$

\section{C6H6-C5H5NCH3 $3 f$}

\begin{tabular}{cccccc} 
Center & \multicolumn{2}{c}{ Atomic } & \multicolumn{2}{c}{ Atomic } & \multicolumn{3}{c}{ Coordinates } \\
Number & Number & Type & X & Y & Z \\
- & Nums & \\
1 & 6 & 0 & 0.905670 & -1.808290 & 0.000000 \\
2 & 6 & 0 & 0.207710 & -1.868290 & 1.212930 \\
3 & 6 & 0 & -1.188050 & -1.988390 & 1.213190 \\
4 & 6 & 0 & -1.885170 & -2.052880 & 0.000000 \\
5 & 6 & 0 & -1.188050 & -1.988390 & -1.213190 \\
6 & 6 & 0 & 0.207710 & -1.868290 & -1.212930 \\
7 & 1 & 0 & 1.992070 & -1.762130 & 0.000000 \\
8 & 1 & 0 & 0.752240 & -1.866880 & 2.154280 \\
9 & 1 & 0 & -1.725610 & -2.079950 & 2.153970 \\
10 & 1 & 0 & -2.964160 & -2.187000 & 0.000000 \\
11 & 1 & 0 & -1.725610 & -2.079950 & -2.153970 \\
12 & 1 & 0 & 0.752240 & -1.866880 & -2.154280 \\
13 & 6 & 0 & 2.298930 & 1.742160 & 0.000000
\end{tabular}




$\begin{array}{rrrrrr}14 & 6 & 0 & 1.591740 & 1.676560 & 1.202080 \\ 15 & 6 & 0 & 0.207710 & 1.556390 & 1.178510 \\ 16 & 6 & 0 & 1.591740 & 1.676560 & -1.202080 \\ 17 & 6 & 0 & 0.207710 & 1.556390 & -1.178510 \\ 18 & 7 & 0 & -0.452390 & 1.512250 & 0.000000 \\ 19 & 1 & 0 & 3.380150 & 1.841360 & 0.000000 \\ 20 & 1 & 0 & 2.098010 & 1.717860 & 2.160480 \\ 21 & 1 & 0 & -0.397620 & 1.507130 & 2.076040 \\ 22 & 1 & 0 & 2.098010 & 1.717860 & -2.160480 \\ 23 & 1 & 0 & -0.397620 & 1.507130 & -2.076040 \\ 24 & 6 & 0 & -1.933280 & 1.468420 & 0.000000 \\ 25 & 1 & 0 & -2.312650 & 2.490620 & 0.000000 \\ 26 & 1 & 0 & -2.264470 & 0.931700 & -0.885140 \\ 27 & 1 & 0 & -2.264470 & 0.931700 & 0.885140 \\ ----------------------------------------------------------------\end{array}$

$\mathrm{HF} / 6-31 \mathrm{G}^{*} \mathrm{E}(\mathrm{RHF})=-516.811637433$

C6H6-C5H5NCH3 3g

\begin{tabular}{|c|c|c|c|c|c|}
\hline \multirow{2}{*}{$\begin{array}{l}\text { Center } \\
\text { Number }\end{array}$} & \multirow{2}{*}{$\begin{array}{l}\text { Atomic } \\
\text { Number }\end{array}$} & \multirow{2}{*}{\multicolumn{2}{|c|}{ Atomic }} & \multicolumn{2}{|c|}{ Coordinates (Angstroms } \\
\hline & & & & & \\
\hline 1 & 6 & 0 & 0.355670 & -1.988961 & 0.000000 \\
\hline 2 & 6 & 0 & -0.339780 & -1.904901 & 1.213280 \\
\hline 3 & 6 & 0 & -1.728650 & -1.726531 & 1.212870 \\
\hline 4 & 6 & 0 & -2.422660 & -1.637181 & 0.000000 \\
\hline 5 & 6 & 0 & -1.728650 & -1.726531 & -1.212870 \\
\hline 6 & 6 & 0 & -0.339780 & -1.904901 & -1.213280 \\
\hline 7 & 1 & 0 & 1.426360 & -2.182321 & 0.000000 \\
\hline 8 & 1 & 0 & 0.192260 & -2.018781 & 2.154980 \\
\hline 9 & 1 & 0 & -2.273380 & -1.697211 & 2.153350 \\
\hline 10 & 1 & 0 & -3.504890 & -1.533761 & 0.000000 \\
\hline 11 & 1 & 0 & -2.273380 & -1.697211 & -2.153350 \\
\hline 12 & 1 & 0 & 0.192260 & -2.018781 & -2.154980 \\
\hline 13 & 6 & 0 & -1.050380 & 1.523219 & 0.000000 \\
\hline 14 & 6 & 0 & -0.339780 & 1.507329 & -1.201540 \\
\hline 15 & 6 & 0 & 1.049180 & 1.485429 & -1.179170 \\
\hline 16 & 6 & 0 & -0.339780 & 1.507329 & 1.201540 \\
\hline 17 & 6 & 0 & 1.049180 & 1.485429 & 1.179170 \\
\hline 18 & 7 & 0 & 1.711630 & 1.478869 & 0.000000 \\
\hline 19 & 1 & 0 & -2.134940 & 1.539339 & 0.000000 \\
\hline 20 & 1 & 0 & -0.847900 & 1.510739 & -2.159390 \\
\hline 21 & 1 & 0 & 1.655290 & 1.477359 & -2.077840 \\
\hline 22 & 1 & 0 & -0.847900 & 1.510739 & 2.159390 \\
\hline 23 & 1 & 0 & 1.655290 & 1.477359 & 2.077840 \\
\hline 24 & 6 & 0 & 3.192650 & 1.505839 & 0.000000 \\
\hline 25 & 1 & 0 & 3.528980 & 2.542679 & 0.000000 \\
\hline 26 & 1 & 0 & 3.553610 & 0.992159 & 0.888230 \\
\hline 27 & 1 & 0 & 3.553610 & 0.992159 & -0.888230 \\
\hline
\end{tabular}


$\mathrm{HF} / 6-31 \mathrm{G} * \mathrm{E}(\mathrm{RHF})=-516.808462435$

C6H6-C5H5NCH3 3h

\begin{tabular}{|c|c|c|c|c|c|}
\hline \multirow{2}{*}{$\begin{array}{l}\text { Center } \\
\text { Number }\end{array}$} & \multirow{2}{*}{$\begin{array}{l}\text { Atomic } \\
\text { Number }\end{array}$} & \multicolumn{2}{|c|}{ Atomic } & \multicolumn{2}{|c|}{ Coordinates (Angstroms) } \\
\hline & & & Type & X & Z \\
\hline 1 & 6 & 0 & -2.613250 & -0.406640 & 1.401440 \\
\hline 2 & 6 & 0 & -2.626790 & 0.806400 & 0.700810 \\
\hline 3 & 6 & 0 & -2.626790 & 0.806400 & -0.700810 \\
\hline 4 & 6 & 0 & -2.613250 & -0.406640 & -1.401440 \\
\hline 5 & 6 & 0 & -2.613250 & -1.619460 & -0.700600 \\
\hline 6 & 6 & 0 & -2.613250 & -1.619460 & 0.700600 \\
\hline 7 & 1 & 0 & -2.640360 & -0.407340 & 2.488000 \\
\hline 8 & 1 & 0 & -2.677640 & 1.746210 & 1.245820 \\
\hline 9 & 1 & 0 & -2.677640 & 1.746210 & -1.245820 \\
\hline 10 & 1 & 0 & -2.640360 & -0.407340 & -2.488000 \\
\hline 11 & 1 & 0 & -2.641980 & -2.560270 & -1.244530 \\
\hline 12 & 1 & 0 & -2.641980 & -2.560270 & 1.244530 \\
\hline 13 & 6 & 0 & 2.210570 & -1.565700 & 0.000000 \\
\hline 14 & 6 & 0 & 0.988810 & -0.886620 & 0.000000 \\
\hline 15 & 6 & 0 & 0.979710 & 0.502760 & 0.000000 \\
\hline 16 & 6 & 0 & 3.396260 & -0.827670 & 0.000000 \\
\hline 17 & 6 & 0 & 3.340690 & 0.561330 & 0.000000 \\
\hline 18 & 7 & 0 & 2.144410 & 1.193340 & 0.000000 \\
\hline 19 & 1 & 0 & 2.238230 & -2.651100 & 0.000000 \\
\hline 20 & 1 & 0 & 0.035860 & -1.405590 & 0.000000 \\
\hline 21 & 1 & 0 & 0.060400 & 1.078820 & 0.000000 \\
\hline 22 & 1 & 0 & 4.366510 & -1.312500 & 0.000000 \\
\hline 23 & 1 & 0 & 4.225350 & 1.187190 & 0.000000 \\
\hline 24 & 6 & 0 & 2.084850 & 2.674420 & 0.000000 \\
\hline 25 & 1 & 0 & 3.097700 & 3.069330 & 0.000000 \\
\hline 26 & 1 & 0 & 1.559590 & 3.004280 & -0.895090 \\
\hline 27 & 1 & 0 & 1.559590 & 3.004280 & 0.895090 \\
\hline
\end{tabular}

$\mathrm{HF} / 6-31 \mathrm{G}^{*} \mathrm{E}(\mathrm{RHF})=-516.814365059$

C6H6-C5H5NCH3 3i

\begin{tabular}{|c|c|c|c|c|c|}
\hline Center & Atomic & & mic & Coordinates & (Angstror \\
\hline Number & Numbe & & Type & $X$ & $\mathrm{Z}$ \\
\hline 1 & 6 & 0 & 1.404561 & -2.904312 & 0.000000 \\
\hline 2 & 6 & 0 & 0.704011 & -2.907362 & 1.213240 \\
\hline 3 & 6 & 0 & -0.697109 & -2.910822 & 1.213240 \\
\hline 4 & 6 & 0 & -1.397659 & -2.911242 & 0.000000 \\
\hline 5 & 6 & 0 & -0.697109 & -2.910822 & -1.213240 \\
\hline 6 & 6 & 0 & 0.704011 & -2.907362 & -1.213240 \\
\hline 7 & 1 & 0 & 2.491231 & -2.926491 & 0.000000 \\
\hline
\end{tabular}




$\begin{array}{ccrrrr}8 & 1 & 0 & 1.248351 & -2.937482 & 2.153810 \\ 9 & 1 & 0 & -1.241289 & -2.943632 & 2.153810 \\ 10 & 1 & 0 & -2.484209 & -2.938752 & 0.000000 \\ 11 & 1 & 0 & -1.241289 & -2.943632 & -2.153810 \\ 12 & 1 & 0 & 1.248351 & -2.937482 & -2.153810 \\ 13 & 6 & 0 & -0.004419 & 0.494038 & 0.000000 \\ 14 & 6 & 0 & -0.005979 & 1.206698 & 1.201590 \\ 15 & 6 & 0 & -0.013350 & 2.595938 & 1.180150 \\ 16 & 6 & 0 & -0.005979 & 1.206698 & -1.201590 \\ 17 & 6 & 0 & -0.013350 & 2.595938 & -1.180150 \\ 18 & 7 & 0 & -0.016500 & 3.259168 & 0.000000 \\ 19 & 1 & 0 & -0.001859 & -0.590602 & 0.000000 \\ 20 & 1 & 0 & -0.006899 & 0.694128 & 2.157620 \\ 21 & 1 & 0 & -0.013510 & 3.203328 & 2.078210 \\ 22 & 1 & 0 & -0.006899 & 0.694128 & -2.157620 \\ 23 & 1 & 0 & -0.013510 & 3.203328 & -2.078210 \\ 24 & 6 & 0 & 0.027710 & 4.740938 & 0.000000 \\ 25 & 1 & 0 & 1.068750 & 5.063598 & 0.000000 \\ 26 & 1 & 0 & -0.481880 & 5.107708 & -0.888050 \\ 27 & 1 & 0 & -0.481880 & 5.107708 & 0.888050 \\ ------------------------------------------------------------------\end{array}$

$\mathrm{HF} / 6-31 \mathrm{G}^{*} \mathrm{E}(\mathrm{RHF})=-516.814073934$

C6H6-C5H5NCH3 3j

\begin{tabular}{cccccc} 
Center & \multicolumn{2}{c}{ Atomic } & \multicolumn{2}{c}{ Atomic } & \multicolumn{3}{c}{ Coordinates } & (Angstroms) \\
Number & Number & Type & X & Y & Z \\
-1 & 6 & 0 & -3.115610 & 0.007930 & 1.400800 \\
2 & 6 & 0 & -3.116240 & 1.221020 & 0.700320 \\
3 & 6 & 0 & -3.116240 & 1.221020 & -0.700320 \\
4 & 6 & 0 & -3.115610 & 0.007930 & -1.400800 \\
5 & 6 & 0 & -3.115610 & -1.204940 & -0.700430 \\
6 & 6 & 0 & -3.115610 & -1.204940 & 0.700430 \\
7 & 1 & 0 & -3.147620 & 0.008180 & 2.487470 \\
8 & 1 & 0 & -3.150350 & 2.161820 & 1.244140 \\
9 & 1 & 0 & -3.150350 & 2.161820 & -1.244140 \\
10 & 1 & 0 & -3.147620 & 0.008180 & -2.487470 \\
11 & 1 & 0 & -3.149390 & -2.145990 & -1.243840 \\
12 & 1 & 0 & -3.149390 & -2.145990 & 1.243840 \\
13 & 6 & 0 & 4.473640 & -0.025160 & 0.000000 \\
14 & 6 & 0 & 3.748870 & -1.220470 & 0.000000 \\
15 & 6 & 0 & 2.360640 & -1.179370 & 0.000000 \\
16 & 6 & 0 & 3.777870 & 1.185140 & 0.000000 \\
17 & 6 & 0 & 2.387130 & 1.178400 & 0.000000 \\
18 & 7 & 0 & 1.710760 & 0.008170 & 0.000000 \\
19 & 1 & 0 & 5.559190 & -0.037990 & 0.000000 \\
20 & 1 & 0 & 4.245620 & -2.184690 & 0.000000 \\
21 & 1 & 0 & 1.738860 & -2.068090 & 0.000000 \\
22 & 1 & 0 & 4.297650 & 2.137100 & 0.000000 \\
23 & 1 & 0 & 1.792460 & 2.084310 & 0.000000
\end{tabular}




\begin{tabular}{rrrrrr}
24 & 6 & 0 & 0.226270 & 0.004590 & 0.000000 \\
25 & 1 & 0 & -0.133970 & 1.029370 & 0.000000 \\
26 & 1 & 0 & -0.128690 & -0.506050 & -0.893150 \\
27 & 1 & 0 & -0.128690 & -0.506050 & 0.893150 \\
\hline
\end{tabular}

$\mathrm{HF} / 6-31 \mathrm{G}^{*} \mathrm{E}(\mathrm{RHF})=-516.812957778$

C6H6-C5H5NCH3 3k

\begin{tabular}{ccccccc} 
Center & \multicolumn{2}{c}{ Atomic } & \multicolumn{2}{c}{ Atomic } & \multicolumn{3}{c}{ Coordinates } & $($ Angstroms) \\
Number & Number & Type & X & Y & Z \\
\hline-1 & 6 & 0 & 1.482520 & -0.287160 & 0.000000 \\
2 & 6 & 0 & 2.654800 & -1.050720 & 0.000000 \\
3 & 6 & 0 & 3.900680 & -0.414650 & 0.000000 \\
4 & 6 & 0 & 3.974420 & 0.982530 & 0.000000 \\
5 & 6 & 0 & 2.802740 & 1.746690 & 0.000000 \\
6 & 6 & 0 & 1.556990 & 1.110110 & 0.000000 \\
7 & 1 & 0 & 0.518120 & -0.790740 & 0.000000 \\
8 & 1 & 0 & 2.600490 & -2.136230 & 0.000000 \\
9 & 1 & 0 & 4.811750 & -1.006450 & 0.000000 \\
10 & 1 & 0 & 4.942680 & 1.475160 & 0.000000 \\
11 & 1 & 0 & 2.862050 & 2.831730 & 0.000000 \\
12 & 1 & 0 & 0.651960 & 1.714490 & 0.000000 \\
13 & 6 & 0 & -2.234290 & -2.188790 & 0.000000 \\
14 & 6 & 0 & -2.231300 & -1.478710 & 1.203100 \\
15 & 6 & 0 & -2.231300 & -0.089120 & 1.180090 \\
16 & 6 & 0 & -2.231300 & -1.478710 & -1.203100 \\
17 & 6 & 0 & -2.231300 & -0.089120 & -1.180090 \\
18 & 7 & 0 & -2.238700 & 0.572990 & 0.000000 \\
19 & 1 & 0 & -2.235270 & -3.274520 & 0.000000 \\
20 & 1 & 0 & -2.224020 & -1.986540 & 2.161560 \\
21 & 1 & 0 & -2.230540 & 0.517150 & 2.078850 \\
22 & 1 & 0 & -2.224020 & -1.986540 & -2.161560 \\
23 & 1 & 0 & -2.230540 & 0.517150 & -2.078850 \\
24 & 6 & 0 & -2.311380 & 2.054290 & 0.000000 \\
25 & 1 & 0 & -3.359490 & 2.353880 & 0.000000 \\
26 & 1 & 0 & -1.809990 & 2.430340 & 0.888910 \\
27 & 1 & 0 & -1.809990 & 2.430340 & -0.888910 \\
-------------------------------------------------------
\end{tabular}

$\mathrm{HF} / 6-31 \mathrm{G} * \mathrm{E}(\mathrm{RHF})=-516.805698256$ 